Board of Governors of the Federal Reserve System

International Finance Discussion Papers

Number 719

February 2002

\title{
INFLATION TARGETING AND NOMINAL INCOME GROWTH TARGETING: WHEN AND WHY ARE THEY SUBOPTIMAL?
}

Jinill Kim and Dale W. Henderson

NOTE: International Finance Discussion Papers are preliminary materials circulated to stimulate discussion and critical comment. References to International Finance Discussion Papers (other than an acknowledgment that the writer has had access to unpublished material) should be cleared with the author or authors. Recent IFDPs are available on the Web at www.federalreserve.gov/pubs/ifdp. 


\title{
INFLATION TARGETING AND NOMINAL INCOME GROWTH TARGETING: WHEN AND WHY ARE THEY SUBOPTIMAL
}

\author{
Jinill Kim and Dale W. Henderson*
}

\begin{abstract}
We derive optimal monetary stabilization rules and compare them to simple rules under both full and partial information. The nominal interest rate is the instrument of monetary policy. Special attention is devoted to inflation targeting and nominal-income-growth targeting. We use an optimizing-agent model of a closed economy which features monopolistic competition in both product and labor markets. A stabilization problem exists because there are one-period nominal contracts, either for wages alone or for both wages and prices, and three shocks that are unknown when contracts are signed. In order to highlight basic theoretical results, we deliberately keep our model simple enough that we can obtain exact solutions. Optimal rules maximize the expected utility of the representative agent subject to the information set of the policymaker. A key result, possibly surprising at first, is that even with monopolistic competition, the optimal full information policy makes the economy mimic the hypothetical equilibrium with flexible prices and wages. We explain why strict versions of inflation targeting, nominal income growth targeting, and other such simple rules are suboptimal under both full and partial information and derive flexible versions that are optimal under certain partial information assumptions. Nominal income growth targeting dominates inflation targeting for plausible parameter values.
\end{abstract}

Keywords: monetary policy, monetary rule, wage contracts, price contracts, full information, partial information.

"The authors would like to thank Robert Usarek for valuable research assistance, Corrado Di Maria and Morten Spange for pointing out errors in previous versions, and Kevin Lansing for helpful comments. Earlier versions of this paper were presented at the University of Kentucky, Aahrus University, the Konstanz Seminar on Monetary Theory and Monetary Policy, the 2002 Winter Meetings of the Econometric Society, the University of Linz, the Central European University, the National Bank of Austria, and the Institute for Advanced Studies in Vienna. Kim's work on this project was supported by a Bankard grant from the University of Virginia. The views in this paper are solely the responsibility of the authors and should not be interpreted as reflecting the views of the Board of Governors of the Federal Reserve System or any other person associated with the Federal Reserve System. The email addresses of the authors are jk9n@virginia.edu and dale.henderson@frb.gov, respectively. 


\section{Introduction}

We derive optimal monetary stabilization rules and compare them to simple rules under both full and partial information. Optimal rules maximize the expected utility of the representative agent subject to the information set of the policymaker. In accordance with the practice of most central banks, we assume throughout that the nominal interest rate, not the money supply, is the instrument of monetary policy. Inflation targeting and nominal income growth targeting receive special attention as in other recent studies. ${ }^{1}$ Inflation targeting is of particular interest because in several countries the monetary policymaking process is referred to as inflation targeting.

We use a model of a closed economy with optimizing firms and households, monopolistic competition in both product and labor markets, and one-period nominal contracts. In this setting and within a range, it is profitable for firms or workers to increase their outputs or labor services in response to increases in demands even though they cannot change prices or wages. A stabilization problem exists because there are three i.i.d. shocks that are unknown when contracts are signed. Agents are 'identical' because contracts are synchronized, so we can consider the utility of the representative agent. This utility can be written as a function of only employment and shocks since consumption is implied by the production function.

Our purpose is to illustrate some basic theoretical results in an easily understandable way. Therefore, we impose enough simplifying assumptions to insure that we can obtain exact analytic solutions. ${ }^{2}$ Of course, monetary rules for actual economies can be evaluated only in more complicated models for which there are no analytic solutions, exact or approximate.

We consider two cases: (1) all wages set in contracts and all prices flexible and (2) all wages and prices set in contracts. ${ }^{3}$ If wages are set in contracts, for some shocks

\footnotetext{
${ }^{1}$ For a comprehensive listing of studies of inflation targeting, see Svensson (1999). For a listing of papers on nominal income targeting written before 1993, see Henderson and McKibbin (1993). For listings of more recent papers, see McCallum and Nelson (1999a), the discussion paper version of McCallum and Nelson (1999b), and Jensen (1999). Frisch and Staudinger (2002) show, as we do, that the ranking of the two rules depends on productivity shocks.

${ }^{2}$ As shown by Woodford (1999), under certain conditions, when shocks have small variances correct welfare rankings are obtained from utility calculations made using the standard linear approximation method developed fully in Rotemberg and Woodford (1998), the discussion paper version of Rotemberg and Woodford (1997). However, in general, even when the variances of shocks are small, incorrect welfare rankings are obtained from the standard method. For example, Kim and Kim (2002) show that in a model of international risk sharing, the standard method implies that welfare can be lower with a complete market than with autarchy.

${ }^{3}$ We can limit our focus to these two cases because, as we show in Henderson and Kim (1999), the outcomes in the third case with price contracts alone are the same as the outcomes in the case of both wage and price contracts for all variables except, of course, for the nominal wage. However, if prices are fixed by staggered contracts instead of by one-period contracts (or by synchronized multiperiod contracts), results depend crucially on whether wages are fixed by contracts or are flexible as shown by Erceg, Henderson, and Levin (2000).

We recognize that it would be very elegant if we could assume that some fraction of wages and prices are set in one-period contracts and the rest are flexible as in, for example, Ohanian, Stockman, and Killian (1995). Then we could investigate the effects of nominal rigidity by varying the fraction of wages and prices set in one-period contracts. Unfortunately, it is not possible to obtain exact
} 
the attractiveness of some simple rules depends crucially on whether prices are also set in contracts. For each case, we determine the optimal rule with commitment when the policymaker has full information, that is, knowledge of the current values of all the shocks.

Although it may seem surprising at first, the optimal rule with nominal contracts generates the unique outcomes for employment that would emerge in equilibrium with full flexibility of wages and prices and given shocks. ${ }^{4}$ With full flexibility, employment does not depend on the price level (neutrality) or inflation (superneutrality) and the mean and variance of employment are independent of monetary policy. Of course, full flexibility employment is below Pareto optimal employment because of the distortions resulting from monopolistic competition, but there is nothing that the policymaker can do to change this fact. ${ }^{5}$ With nominal contracts, monetary policy can affect both the mean and variance of employment. However, expected utility with nominal contracts is always less than or equal to expected utility with full flexibility. That is, it is not welfare improving to raise expected employment above the full-flexibility level toward the level it would have without distortions. Thus, the policymaker's best response is to replicate full flexibility employment, and this response is feasible with full information. ${ }^{6}$

For us 'targeting' a variable means either total or partial stabilization of that variable and 'strict'('flexible') targeting means total (partial) offsetting of deviations of a variable from a target value. The policymaker must target a nominal variable (either inflation or nominal income) if there is to be a determinate rational expectations equilibrium; that is, there must be a 'nominal anchor.' If the policymaker responds to deviations of two variables-for example, inflation and output-from target values, we say it is targeting a combination of those variables. ${ }^{7}$ We show that inflation targeting, nominal income growth targeting, and some other simple rules are suboptimal with full information. Among suboptimal rules, nominal income growth targeting dominates inflation targeting for plausible parameter values.

A policymaker might use a simple rule based on a limited subset of variables

solutions under this assumption in our one sector model.

${ }^{4}$ Obstfeld and Rogoff (2000) have shown that under certain assumptions, optimal stabilization policy mimics the full flexibility equilibrium even in the presence of distortions resulting from monopolistic competition. We replicate this result in a closed-economy setting.

${ }^{5}$ As stated above,we assume that the policymaker must commit itself to a monetary rule. In the absence of this assumption, the policymaker would have an incentive to create surprise inflation in order to raise output and employment above their full flexibility levels because of the distortions arising from monopolistic competition. The inflation rate would be indeterminate because under our assumptions inflation does not enter the objective function of the policymaker which is the utility function of the representative agent.

${ }^{6}$ However, replication of the full flexibility equilibrium may not represent the upper bound in the presence of externalities. For example, the policymaker can do better than replication of the full flexibility equilibrium in the model of Koenig (1996) which incorporates nominal stickiness and an externality.

${ }^{7}$ We recognize that some authors use the term "flexible inflation targeting" to refer to any rule in which the monetary authorities respond to inflation deviations no matter whether they respond to other deviations or not. However, we think that the terminology in the text is clearer. 
because it does not have information on other variables. ${ }^{8}$ We determine the optimal rules under alternative assumptions about partial information. Under these assumptions, the policymaker knows the current values of some or all of the variables determined by the model (inflation, output growth, and employment growth) and can infer something but not necessarily everything about the current values of some of the shocks. We show that strict inflation targeting, strict nominal income growth targeting, and some other simple rules are suboptimal with partial information. Also, flexible versions of these same rules are suboptimal except possibly under the very restrictive assumption that inflation, nominal income growth, or some other limited set of variables is the only information available to the authorities.

Our paper is closely related to two sets of recent studies. The first set shares our objective: evaluation of alternative monetary policies in a setting similar to ours. ${ }^{9}$ Also, in this set the interest rate is often the instrument of monetary policy. ${ }^{10}$ However, all the authors use approximate solutions, and most use simulations because obtaining exact analytic solutions is not possible in their models. We supplement these studies by obtaining exact analytic solutions in a simpler model.

The second set shares our modeling strategy: using a simple model with an exact analytic solution. ${ }^{11}$ However, the setup differs from ours in all these papers, and the objective differs in all but one. As regards setup, for us the nominal interest rate is the instrument of policy, and it responds to shocks or changes in variables. Although we adopt the conventional assumption that the period utility function is additively separable, we consider a limiting case in which real money balances are constant except for response to a shock. In this case, variation in the nominal interest rate does not generate welfare costs associated with variation in real money balances. In the second set, the money supply is the instrument of policy and in all but one of the studies it is either kept constant or is varied so as to fix the exchange rate. Some authors allow for variation in real money balances associated with variation in the nominal interest rate but simply assume that the welfare costs associated with this variation are small enough to be neglected. Others assume that consumption and real balances enter utility as logarithms and that the growth rate of the money supply is i.i.d. In the latter situation, the nominal interest rate remains constant. As regards objectives, we evaluate alternative monetary stabilization rules in a stochastic model with three shocks. One two-country study considers the utility effects of alternative money supply responses to a productivity shock, but all the other exact-solution

${ }^{8}$ Also a policymaker might choose to follow a simple rule if it believes that it would be too difficult to explain a more complicated rule to the public.

${ }^{9}$ This set includes Ireland (1997), Goodfriend and King (1997), Rotemberg and Woodford (1997), Rotemberg and Woodford (1999), King and Wolman (1999), and Henderson and Kim (2001).

${ }^{10}$ Among the studies cited in the preceding footnote, Henderson and Kim (2001), King and Wolman (1999), Rotemberg and Woodford (1997), and Rotemberg and Woodford (1999) assume that the interest rate is the instrument of policy.

${ }^{11}$ Benassy (1995), Corsetti and Pesenti (2001), and Obstfeld and Rogoff (1998) have discovered closely related sets of assumptions under which exact analytic solutions can be obtained in closed-economy, stochastic models; two-country, perfect foresight models; and two-country, stochastic models, respectively. In Henderson and Kim (1999) and this paper, we use many of the same assumptions. 
studies focus either on the utility effects of a one-time increase in the money supply in a perfect foresight model, on the implications of an exogenous money supply process in a stochastic model, or on a utility comparison of fixed and flexible exchange rates in a stochastic model. ${ }^{12}$

The rest of this paper is organized into five more sections. Section 2 is a description of our model. We devote section 3 to the benchmark version with full flexibility of wages and prices. In sections 4 and 5 , respectively, we analyze alternative monetary policy rules in versions with wage contracts and flexible prices and with both wage and price contracts. Section 6 contains our conclusions.

\section{The Model}

In this section we describe our model. We discuss the behavior of firms, households, and the government in successive subsections.

\section{$2.1 \quad$ Firms}

A continuum of 'identical' monopolistically competitive firms is distributed on the unit interval, $f \in[0,1]$. With no price contracts, firms set their prices for period $t$ based on period $t$ information. With one-period price contracts, firms set prices for period $t+1$ based on period $t$ information and agree to supply whatever their customers demand at those prices. In either case, the problem of firm $f$ in period $t$ is to find the

$$
\max _{\left\{\mathrm{P}_{f, t+j}\right\}} \mathcal{E}_{t} \tilde{\delta}_{t, t+j}\left(s_{P} \mathrm{P}_{f, t+j} \mathrm{Y}_{f, t+j}-W_{t+j} L_{f, t+j}\right)
$$

where capital letters without serifs represent choice variables of individual firms or households and capital letters with serifs represent indexes that include all firms or households. The subscript $j$ takes on the value 0 if there are no price contracts and the value 1 if there are price contracts. In period $t$, firm $f$ sets the price $\mathrm{P}_{f, t+j}$. In period $t+j$ in a given state, firm $f$ produces output $\mathrm{Y}_{f, t+j}$, and employs the amount $L_{f, t+j}$ of a labor index $L_{t+j}$ for which it pays the wage index $W_{t+j}$ per unit:

$$
L_{t+j}=\int_{0}^{1} L_{f, t+j} d f=\left(\int_{0}^{1} \mathrm{~L}_{h, t+j}^{\frac{1}{\theta_{W}}} d h\right)^{\theta_{W}} \quad W_{t+j}=\left(\int_{0}^{1} \mathrm{~W}_{h, t+j}^{\frac{1}{1-\theta_{W}}} d h\right)^{1-\theta_{W}}
$$

where $\mathrm{L}_{h, t+j}$ is the amount of labor supplied by household $h$ in period $t+j, \mathrm{~W}_{h, t+j}$ is the wage charged by household $h$ in period $t+j$, and $\theta_{W}>1$. Firm $f$ chooses quantities of $\mathrm{L}_{h, t+j}$ to minimize the cost of producing a unit of $L_{f, t+j}$ given the $\mathrm{W}_{h, t+j}$, and $W_{t+j}$ is the minimum cost. All firms receive an ad valorem output subsidy, $s_{P}$. $\tilde{\delta}_{t, t+j}$ is a stochastic discount factor, the price of a claim to one dollar delivered in a particular state in period $t+j$ divided by the probability of that state. We use

\footnotetext{
${ }^{12}$ The studies with stochastic models not already cited are Obstfeld and Rogoff (2000), Devereux and Engel (1998), and Engel (1999).
} 
$\mathcal{E}_{t}$ to indicate an expectation taken over the states in period $t+j$ based on period $t$ information. The production function of firm $f$ is ${ }^{13}$

$$
\mathrm{Y}_{f, t+j}=\frac{L_{f, t+j}^{(1-\alpha)} X_{t+j}}{1-\alpha}
$$

where $X_{t+j}$ is a productivity shock that hits all firms, and $x_{t+j}=\ln X_{t+j} \backsim N\left(0,2 \sigma_{x}^{2}\right)$. An expression for $L_{f, t+j}$ is obtained by inverting this production function.

Relative demand for output of firm $f$ is a decreasing function of its relative price:

$$
\frac{\mathrm{Y}_{f, t+j}}{Y_{t+j}}=\left(\frac{\mathrm{P}_{f, t+j}}{P_{t+j}}\right)^{-\frac{\theta_{P}}{\theta_{P}-1}}
$$

where $\theta_{P}>1$. In equation (4), $Y_{t+j}$ is an index made up of the output of all firms and $P_{t+j}$ is a price index which is the price of a unit of the output index:

$$
Y_{t+j}=\int_{0}^{1} Y_{h, t+j} d h=\left(\int_{0}^{1} \mathrm{Y}_{f, t+j}^{\frac{1}{\theta_{P}}} d f\right)^{\theta_{P}} \quad P_{t+j}=\left(\int_{0}^{1} \mathrm{P}_{f, t+j}^{\frac{1}{1-\theta_{P}}} d f\right)^{1-\theta_{P}}
$$

where $Y_{h, t+j}$ is the amount of the output index purchased by household $h$ in period $t+j$. Household $h$ chooses quantities of $\mathrm{Y}_{f, t+j}$ to minimize the cost of producing a unit of $Y_{h, t+j}$ given the $\mathrm{P}_{f, t+j}$, and $P_{t+j}$ is the minimum cost.

To maximize profits, a firm must set its price so that expected discounted marginal revenue equals expected discounted marginal cost:

$$
s_{P}\left(\frac{1}{\theta_{P}-1}\right) \mathcal{E}_{t}\left(\tilde{\delta}_{t, t+j} \mathrm{Y}_{f, t+j}\right)=\left(\frac{\theta_{P}}{\theta_{P}-1}\right) \mathcal{E}_{t}\left(\frac{\tilde{\delta}_{t, t+j} W_{t+j} L_{f, t+j}^{\alpha} \mathrm{Y}_{f, t+j}}{\mathrm{P}_{f, t+j} X_{t+j}}\right)
$$

Since firms are identical,

$$
L_{f,+j}=L_{+j} \quad \mathrm{Y}_{f,+j}=Y_{+j} \quad P_{f,+j}=P_{+j}
$$

where we omit $t$ subscripts in the rest of this subsection for simplicity. Therefore, the equalities in (7) imply that the 'aggregate production function' and 'aggregate price equation' are, respectively,

$$
\begin{gathered}
Y_{+j}=\frac{L_{+j}^{(1-\alpha)} X_{+j}}{1-\alpha} \\
s_{P} \mathcal{E}\left(\tilde{\delta}_{+j} Y_{+j}\right)=\theta_{P} \mathcal{E}\left(\frac{\tilde{\delta}_{+j} W_{+j} L_{+j}^{\alpha} Y_{+j}}{P_{+j} X_{+j}}\right)
\end{gathered}
$$

\footnotetext{
${ }^{13}$ That is, we assume for simplicity that there are no factors of production other than labor and no fixed costs. Kim (2002) shows that our formulation can be viewed as a model with capital in which the marginal adjustment cost for the first unit of net investment approaches infinity. Kim (1997) explores the implications of allowing for fixed costs.
} 
When $j=0$ so that period $t$ prices are set on the basis of period $t$ information, the aggregate price equation (9) can be rewritten as

$$
\left(\frac{s_{P}}{\theta_{P}}\right) \frac{X}{L^{\alpha}}=\frac{W}{P}
$$

which states that $P$ must be chosen so that the marginal value product of labor (the gross subsidy rate over the markup parameter times the marginal product of labor) equals the real wage.

\subsection{Households}

A continuum of 'identical' households is distributed on the unit interval, $h \in[0,1]$. With no wage contracts, households set their wages for period $t$ based on period $t$ information, but with wage contracts they set their wages for period $t+1$ based on period $t$ information. The problem of household $h$ in period $t$ is to find the

$$
\max _{\left\{C_{h, \tau}, M_{h, \tau}, B_{h, \tau}, B_{h, \tau}^{g}, \mathrm{~W}_{h, \tau+j}\right\}} \mathcal{E}_{t} \sum_{\tau=t}^{\infty} \beta^{\tau-t} \mathbb{U}\left(C_{h, \tau}, \frac{M_{h, \tau}}{P_{\tau}}, \mathrm{L}_{h, \tau}\right)
$$

where

$$
\mathbb{U}\left(C_{h, \tau}, \frac{M_{h, \tau}}{P_{\tau}}, \mathrm{L}_{h, \tau}\right)=\left[\frac{C_{h, \tau}^{1-\rho}}{1-\rho}+\frac{\iota_{0}\left(\frac{M_{h, \tau}}{P_{\tau} V_{\tau}}\right)^{1-\iota}}{1-\iota}-\frac{\chi_{0} \mathrm{~L}_{h, \tau}^{1+\chi}}{Z_{\tau}(1+\chi)}\right] U_{\tau}
$$

subject to

$$
\begin{aligned}
& C_{h, \tau}= \frac{s_{W} \mathrm{~W}_{h, \tau} \mathrm{L}_{h, \tau}}{P_{\tau}}+\frac{R_{\tau}}{P_{\tau}}-T_{h, \tau} \\
&-\frac{M_{h, \tau}-M_{h, \tau-1}+\boldsymbol{\delta}_{\tau, \tau+\mathbf{1}} \mathrm{B}_{\mathbf{h}, \tau}-B_{h, \tau-1}+B_{h, \tau}^{g}-I_{\tau-1} B_{h, \tau-1}^{g}}{P_{\tau}} \\
& \frac{\mathrm{L}_{h, \tau}}{L_{\tau}}=\left(\frac{\mathrm{W}_{h, \tau}}{W_{\tau}}\right)^{-\frac{\theta_{W}}{\theta_{W}-1}}
\end{aligned}
$$

According to equation (12), the period utility $(\mathbb{U})$ of household $h$ depends positively on its consumption $\left(C_{h, \tau}\right)$ and the ratio of its real balances $\frac{M_{h, \tau}}{P_{\tau}}$ to a shock $\left(V_{\tau}\right)$, and negatively on its labor supply $\left(\mathrm{L}_{h, \tau}\right) .{ }^{14}$ The period budget constraint, equation (13), states that consumption must equal disposable income minus asset accumulation. Each household is a monopolistically competitive supplier of its unique labor input.

\footnotetext{
${ }^{14}$ If the first term of the utility function has the form $\frac{C_{h, \tau}^{1-\rho}-1}{1-\rho}$, it has $\ln C_{h, \tau}$ as a limit as $\rho$ approaches 1. For simplicity and comparability with other studies, we use the form in the text. We can also obtain exact solutions if we use the form in the footnote, and these solutions have the same qualitative properties as those obtained using the form in the text.
} 
Relative demand for labor of household $h$ is a decreasing function of its relative wage as shown in equation (14)

In period $\tau$, household $h$ chooses its consumption and its holdings of money, $M_{h, \tau}$. Household $h$ also chooses its wage rate in period $\tau+j, \mathbf{W}_{h, \tau+j}$, and agrees to supply however many units of its labor, $\mathrm{L}_{h, \tau+j}$, firms want at this wage where the subscript $j$ takes on the value 0 if there are no wage contracts and the value 1 if there are wage contracts. In addition, in period $\tau$, household $h$ chooses its holdings of claims to a unit of currency in the various states in period $\tau+1$. Each element in the infinite-dimensional vector $\boldsymbol{\delta}_{\tau, \tau+\mathbf{1}}$ represents the price of an asset that will pay one unit of currency in a particular state of nature in the subsequent period, while the corresponding element of the vector $\mathbf{B}_{\mathbf{h}, \tau}$ represents the quantity of such claims purchased by the household. ${ }^{15}$ The scalar variable $B_{h, \tau-1}$ represents the value of the households's claims given the current state of nature. Household $h$ also chooses its holding of government bonds $B_{h, \tau}^{g}$, which pay $I_{\tau}$ units of currency in every state of nature in period $\tau+1$. Household $h$ receives an aliquot share, $R_{\tau}$, of aggregate profits and pays lump sum taxes, $T_{h, \tau} \cdot{ }^{16}$ All households receive an ad valorem labor subsidy, $s_{W}$. There are goods demand, $U_{\tau}$, money demand, $V_{\tau}$, and labor supply shocks, $Z_{\tau}$, that hit all consumers. We assume that the shocks $U_{\tau}, V_{\tau}$, and $Z_{\tau}$ have lognormal distributions. ${ }^{17}$ We impose the restrictions that $0<\beta<1, \rho \geq 0$, and $\chi \geq 0 . \mathcal{E}_{t}$ indicates an expectation over the various states in period $\tau$ based on period $t$ information.

The first order conditions for household $h$ for consumption, nominal balances, contingent claims, and government bonds for period $t$ and for the nominal wage in period $t+j, j=0$ or 1 are obtained by substituting equation (14) into equation (13), constructing a Lagrangian expression with a multiplier $\eta_{h, \tau}$ associated with the period budget constraint for state in period $\tau$, and differentiating .

$$
\begin{gathered}
\frac{U_{t}}{C_{h, t}^{\rho}}=\eta_{h, t} \\
\frac{\iota_{0} U_{t}}{\left(\frac{M_{h, t}}{P_{t} V_{t}}\right)^{\iota}} \frac{1}{P_{t} V_{t}}=\frac{\eta_{h, t}}{P_{t}}-\beta \mathcal{E}_{t}\left(\frac{\eta_{h, t+1}}{P_{t+1}}\right) \\
\frac{\tilde{\delta}_{t, t+1} \eta_{h, t}}{P_{t}}=\frac{\beta \eta_{h, t+1}}{P_{t+1}}
\end{gathered}
$$

${ }^{15}$ Let $\delta_{\tau, \tau+1}(\zeta)$ represent the element of $\boldsymbol{\delta}_{\boldsymbol{\tau}, \boldsymbol{\tau}+\mathbf{1}}$ that corresponds to state $\zeta$ in time $\tau+1$. Then $\delta_{\tau, \tau+1}(\zeta)=\tilde{\delta}_{\tau, \tau+1}(\zeta) \operatorname{Pr}(\zeta)$, where $\operatorname{Pr}(\zeta)$ represents the probability at time $\tau$ of state $\zeta$ in time $\tau+1$.

${ }^{16}$ These equal shares exhaust aggregate profits:

$$
\int_{0}^{1} R_{s} d h=\int_{0}^{1}\left(s_{P} \mathrm{P}_{f, s} \mathrm{Y}_{f, s}-W_{s} L_{f, s}\right) d f
$$

${ }^{17}$ That is, we assume that $u_{s}=\log U_{s} \backsim N\left(0,2 \sigma_{u}^{2}\right), v_{s}=\log V_{s} \backsim N\left(0,2 \sigma_{v}^{2}\right)$, and $z_{s}=\log Z_{s} \backsim$ $N\left(0,2 \sigma_{z}^{2}\right)$. 


$$
\begin{aligned}
\frac{\eta_{h, t}}{P_{t}} & =\beta I_{t} \mathcal{E}_{t}\left(\frac{\eta_{h, t+1}}{P_{t+1}}\right) \\
s_{W}\left(\frac{\theta_{W}}{\theta_{W}-1}-1\right) \mathcal{E}_{t}\left(\frac{\eta_{h, t+j} \mathrm{~L}_{h, t+j}}{P_{t+j}}\right) & =\chi_{0}\left(\frac{\theta_{W}}{\theta_{W}-1}\right) \mathcal{E}_{t}\left(\frac{\left(\mathrm{L}_{h, t+j}\right)^{\chi} \mathrm{L}_{h, t+j} U_{t+j}}{\mathrm{~W}_{h, t+j} Z_{t+j}}\right)
\end{aligned}
$$

In order to make it possible to obtain exact analytic solutions in which the nominal interest rate can vary, we assume that $\iota \rightarrow \infty$. Under this assumption, the first order conditions (15), (16), and (18) imply

$$
\frac{M_{h, t}}{P_{t} V_{t}}=\lim _{\iota \rightarrow \infty}\left[\left(\frac{I_{t}}{I_{t}-1}\right) \frac{\iota_{0} C_{h, t}^{\rho}}{V_{t}}\right]^{\frac{1}{\iota}}=1
$$

where $I_{t}$ represents the gross nominal interest rate, one plus the nominal interest rate. $I_{t}$ must be equal to one over the cost of acquiring claims to one unit of currency in every state of nature in period $t+1$ :

$$
I_{t}=\frac{1}{\int \delta_{t, t+1}}
$$

where the integral is over the states of nature in period $t+1$. Hereafter, we refer to the gross nominal interest rate as the interest rate. According to equation (20), it is optimal for household $h$ to keep its real money holdings constant except for response to a shock. ${ }^{18}$ Furthermore, under the assumption that $\iota \rightarrow \infty$, the period utility function relevant for scoring outcomes becomes

$$
\mathbb{U}\left(C_{h, t}, \mathrm{~L}_{h, t}\right)=\left(\frac{C_{h, t}^{1-\rho}}{1-\rho}-\frac{\chi_{0} \mathrm{~L}_{h, t}^{1+\chi}}{Z_{t}(1+\chi)}\right) U_{t}
$$

since

$$
\lim _{\iota \rightarrow \infty}\left(\frac{\iota_{0}\left[\left(\frac{I_{t}}{I_{t}-1}\right) \frac{\iota_{0} C_{h, t}^{\rho}}{V_{t}}\right]^{\frac{1-\iota}{\iota}} U_{t}}{1-\iota}\right)=0
$$

The first order conditions for household $h$ have implications for relationships among aggregate variables. Since households are identical,

$$
C_{h}=C, \mathrm{~L}_{h}=L, W_{h}=W, T_{h}=T, M_{h}=M, B_{h}=B, \eta_{h}=\eta
$$

where from here on we omit all $t$ subscripts for simplicity. Eliminating $\eta$ and $\eta_{+1}$ using the condition that in each period in each state

$$
\frac{U_{+j}}{C_{+j}^{\rho}}=\eta_{+j}
$$

\footnotetext{
${ }^{18}$ If $\iota$ remains finite, then money demand depends on both $I_{t}$ and $I_{t}-1$, so it is not possible to obtain an exact solution.
} 
yields the contingent claims pricing equations, the consumption Euler equation, the aggregate wage setting equation, and the money market equilibrium condition:

$$
\begin{aligned}
& \tilde{\delta}_{+1}\left(\frac{U}{P C^{\rho}}\right)=\beta\left(\frac{U_{+1}}{P_{+1} C_{+1}^{\rho}}\right) \\
& \frac{U}{P C^{\rho}}=\beta I \mathcal{E}\left(\frac{U_{+1}}{P_{+1} C_{+1}^{\rho}}\right) \\
& \theta_{W} \chi_{0} \mathcal{E}\left(\frac{L_{+j}^{1+\chi} U_{+j}}{W_{+j} Z_{+j}}\right)=s_{W} \mathcal{E}\left(\frac{L_{+j} U_{+j}}{P_{+j} C_{+j}^{\rho}}\right) \\
& M=P V
\end{aligned}
$$

There are as many contingent claims pricing equations as there are states in period +1 . Each contingent claims pricing equation takes the form of equation (26) which states that the price of a claim to a unit of purchasing power in a given state in period +1 must be such that utility forgone in order to purchase such a claim divided by the probability of that state is equal to the discounted utility of a unit purchasing power in that state.

The consumption Euler equation (27) states that $C$ must be chosen so that the utility forgone by not spending a dollar on consumption today equals the discounted expected utility of investing that dollar in a riskless security and spending it on consumption tomorrow.

According to the wage setting equation (28), the wage should be increased until the expected reduction in the disutility of labor just equals the expected loss in utility from consumption resulting from the reduction in wage income. When $j=0$ so that consumers set their wages on the basis of current information, condition (28) can be rewritten as

$$
\left(\frac{s_{W}}{\theta_{W}}\right) \frac{W}{P}=\frac{\chi_{0} L^{\chi} C^{\rho}}{Z}
$$

Equation (30) states that $W$ must be chosen so that the marginal return from work (the subsidy-adjusted real wage divided by the wage markup) must equal the marginal rate of substitution of consumption for labor.

\subsection{Government}

The government budget constraint is

$$
\frac{M-M_{-1}+B^{g}-I_{-1} B_{-1}^{g}}{P}=G+\left(s_{P}-1\right) Y+\left(s_{W}-1\right) \frac{W}{P} L-T
$$

where $G$ is real government spending. We impose simple assumptions about the paths of government spending, interest payments, subsidy payments, and taxes under 
which we can study alternative monetary policy reaction functions. ${ }^{19}$. In particular, we assume that the government budget is balanced period by period and that real government spending is always zero, so the government budget constraint becomes ${ }^{20}$

$$
\frac{\left(I_{-1}-1\right) B_{-1}^{g}}{P}+\left(s_{P}-1\right) Y+\left(s_{W}-1\right) \frac{W}{P} L-T=0
$$

We assume that the government follows a monetary policy rule in the $\operatorname{class}^{21}$

$$
I=\beta^{-1} \Pi^{\lambda_{\Pi}} \Gamma^{\lambda_{\Gamma}} \Upsilon^{\lambda_{\Upsilon}} \Lambda^{\lambda_{\Lambda}} U^{\lambda_{U}} X^{\lambda_{X}} Z^{\lambda_{Z}}
$$

where

$$
\Pi=\frac{P}{P_{-1}}, \quad \Gamma=\frac{Y}{Y^{*}}, \quad \Upsilon=\frac{Y}{Y_{-1}} \quad \Lambda=\frac{L}{L_{-1}}
$$

For rules in this class, either the inflation rate or nominal income is the "nominal anchor. ${ }^{22}$ As we show below, $\lambda_{\Pi}$ must exceed unity in order for the price level to be determined. ${ }^{23}$ We derive the optimal $\lambda_{j}$, the ones that maximize expected utility. We also consider some alternative values of the $\lambda_{j}$.

\footnotetext{
${ }^{19}$ Assumptions about the paths of government spending and taxes have implications for which monetary policies are feasible and for the effects of different feasible monetary policies. For up to date discussions of the interaction between monetary and fiscal policy and citations of other recent contributions see Canzoneri, Cumby, and Diba (2001) and Benhabib, Schmitt-Grohe, and Uribe (2001).

${ }^{20}$ We assume a monetary policy reaction function with target inflation rate of zero but we could just as well have chosen a nonzero target inflation rate. Note that with our utility function there are no welfare costs of inflation.

${ }^{21}$ For completeness we have included a money demand shock, $V$, in the money market equilibrium condition. Even if we included $V$ in the monetary rule, it would be optimal not to respond to movements in $V$. The policymaker would respond to movements in $V$ if it responded to movements in the level or the growth rate of the money supply. Allowing response to the level is straightforward but does not yield much additional insight. Allowing response to the growth rate complicates the analysis considerably. Therefore, we do not allow such responses in this paper. However, Henderson and Kim (1999) allow response to the level, Kim (2000) allows response to the growth rate, and Henderson and Kim (2001) allow response to both.

${ }^{22}$ In Henderson and Kim (1999) we study rules with responses to levels of variables, such as
}

$$
I=\beta^{-1}\left(\frac{P}{\bar{P}}\right)^{\lambda_{P}} \Gamma^{\lambda_{\Gamma}}\left(\frac{Y}{\bar{Y}}\right)^{\lambda_{Y}}\left(\frac{L}{\bar{L}}\right)^{\lambda_{L}} U^{\lambda_{U}} X^{\lambda_{X}} Z^{\lambda_{Z}}
$$

using a model with exact solutions, where $\bar{P}, \bar{Y}$, and $\bar{L}$ are target levels of $P, Y$, and $L$, respectively. In Henderson and Kim (2001) we compare rules in which the interest rate responds to levels with rules in which it responds to changes in a model with approximate solutions. In models with oneperiod contracts, targeting the price level, output, or nominal income has the same effects on real variables as targeting inflation, output growth, and nominal income growth, respectively, whenever $\bar{P}=P_{t-1}$ and $\bar{Y}=Y_{-1}$; the target changes for inflation, output growth,and nominal income growth are zero; and $\lambda_{P}=\lambda_{\Pi}$ and $\lambda_{Y}=\lambda_{\Upsilon}$. However, the effects on expected inflation and, therefore, nominal interest rates are different under levels and changes targeting whenever the current price level is different from $\bar{P}=P_{t-1}$. With levels targeting agents expect that the price level will return to $\bar{P}=P_{t-1}$ but with changes targeting agents expect that it will remain at it current level.

${ }^{23}$ Under levels targeting $\lambda_{P}$ must exceed zero in order for the price level to be determined. 


\section{$3 \quad$ Full Flexibility}

We consider three versions of our model. To establish a benchmark, we begin by considering the version with full flexibility of wages and prices, denoted by $W^{F} P^{F}$ where the superscript $F$ stands for the word 'flexible.'

\subsection{Solution}

In each version of the model six equations are used to determine the equilibrium values of the variables. With $W^{F} P^{F}$, the forms of these six equations are

$$
\begin{gathered}
Y=\frac{L^{\tilde{\alpha}} X}{\tilde{\alpha}} \\
\Pi=\frac{L^{\alpha} \Omega}{X} \frac{\theta_{P}}{s_{P}} \\
\frac{\theta_{W}}{s_{W}} \frac{\chi_{0} L^{\tilde{\chi}} U}{\Omega Z}=\frac{L U}{Y^{\rho} \Pi} \\
\beta I \mathcal{E}\left(\frac{U_{+1}}{Y_{+1}^{\rho} \Pi_{+1}}\right)=\frac{U}{Y^{\rho}} \\
I=\beta^{-1} \Pi^{\lambda_{\Pi}} \Gamma^{\lambda_{\Gamma}} \Upsilon^{\lambda_{\Upsilon}} \Lambda^{\lambda_{\Lambda}} U^{\lambda_{U}} X^{\lambda_{X}} Z^{\lambda_{Z}} \\
M=P V
\end{gathered}
$$

where we have imposed the equilibrium conditions that $C=Y$ and $C_{+1}=Y_{+1}$ and where $\tilde{\alpha}=1-\alpha$ and $\tilde{\chi}=1+\chi$. Recall that $\Pi=\frac{P}{P_{-1}}$ is the gross inflation rate. As will become evident, it is useful to define the variable $\Omega=\frac{W}{P_{-1}}$ which represents today's nominal wage divided by yesterday's price level. With $W^{F} P^{F}$, both wages $(\Omega)$ and prices $(\Pi)$ are set after the shocks are known, and the only expected magnitudes are in the demand equation.

The solutions for selected variables are shown in Table 1. Substituting the solutions for these variables into the equations of the model yields the solutions for the other variables. ${ }^{24}$

Substituting the production and price equations into the wage equation and solving yields the solution for $L$, and substituting the solution for $L$ into the production function yields the solution for $Y$ :

$$
\begin{gathered}
L^{*}=\left(\frac{H}{D} X^{-\tilde{\rho}} Z\right)^{\frac{1}{J}} \\
Y^{*}=\left(\frac{1}{\tilde{\alpha}}\right)\left(\frac{H}{D}\right)^{\frac{\tilde{\alpha}}{J}} X^{\frac{\tilde{\chi}}{J}} Z^{\frac{\tilde{\alpha}}{\mathcal{J}}} \\
H=\frac{\tilde{\alpha}^{\rho}}{\chi_{0}}, \quad D=\frac{\theta_{W} \theta_{P}}{s_{W} s_{P}}, \quad J=\tilde{\alpha} \tilde{\rho}+\tilde{\chi}
\end{gathered}
$$

where $\tilde{\rho}=\rho-1$. $D$ is an index of distortion. Recall that $\theta_{W}$ and $\theta_{P}$ are markups which must exceed one $\left(1<\theta_{W}, \theta_{P}<\infty\right)$. $s_{W}$ and $s_{P}$ are gross subsidy rates which

\footnotetext{
${ }^{24}$ The properties of log normal distributions used in this paper are summarized in the Appendix.
} 
are greater than one when production and employment are being subsidized and less than one when these activities are being taxed. We assume throughout that $D$ is greater than one. Note that for given gross subsidy rates, the index of distortion rises with the product of the markups. The index is equal to one when the product of the markups is equal to the product of the gross subsidy rates.

To solve for the inflation rate we use the method of undetermined coefficients. Suppose that $\Pi$ takes the form

$$
\Pi^{*}=\Phi Y_{-1}^{\phi_{Y}} L_{-1}^{\phi_{L}} U^{\phi_{U}} X^{\phi_{X}} Z^{\phi_{Z}}
$$

We find $\Phi, \phi_{U}, \phi_{X}$, and $\phi_{Z}$ by beginning with the demand equation and eliminating $Y, Y_{+1}$, and $Y^{*}$ using the solution for $Y^{*}$, eliminating $\Pi$ using the conjectured solution (36), and eliminating $I$ using the rule equation to obtain

$$
\begin{aligned}
& -\left(\lambda_{\Pi}-1\right) \ln \Phi-\lambda_{\Pi} \phi_{Y} y_{-1}-\lambda_{\Pi} \phi_{L} l_{-1}-\lambda_{\Pi}\left(\phi_{U} u+\phi_{X} x+\phi_{Z} z\right) \\
& =\left(\lambda_{\Upsilon}-\phi_{Y}\right) \ln \left(\tilde{\alpha}^{-1}\left(\frac{H}{D}\right)^{\frac{\tilde{\alpha}}{J}}\right)+\left(\frac{\lambda_{\Lambda}-\phi_{L}}{J}\right) \ln \left(\frac{H}{D}\right)-\lambda_{\Upsilon} y_{-1}-\lambda_{\Lambda} l_{-1}+\ln \mathcal{E} Q_{1} \\
& +\left(\lambda_{U}-1\right) u+\left(\frac{\lambda_{X} J+\tilde{\chi}\left(\lambda_{\Upsilon}-\phi_{Y}+\rho\right)-\tilde{\rho}\left(\lambda_{\Lambda}-\phi_{L}\right)}{J}\right) x+\left(\frac{\lambda_{Z} J+\tilde{\alpha}\left(\lambda_{\Upsilon}-\phi_{Y}+\rho\right)+\left(\lambda_{\Lambda}-\phi_{L}\right)}{J}\right) z \\
& H=\frac{\tilde{\alpha}^{\rho}}{\chi_{0}}, \quad D=\frac{\theta_{W} \theta_{P}}{s_{W} s_{P}}, \quad J=\tilde{\alpha} \tilde{\rho}+\tilde{\chi}, \quad Q_{1}=U_{+1}^{1-\phi_{U}} X_{+1}^{-\phi_{X}-\frac{\rho \tilde{\chi}}{J}} Z_{+1}^{-\phi_{Z}-\frac{\rho \tilde{\alpha}}{J}}
\end{aligned}
$$

where lower case letters represent logarithms. If equation (37) is to hold for all $U, X$, and $Z$, it must be that the $\phi_{j}$ and $\Phi$ take on the values given in Table 1 in the Appendix and that $\Pi^{*}$ and $\Omega^{*}$ have the solutions given in the same Table. We assume that $\lambda_{\Pi}$ exceeds positive one so it is possible to solve for $\Phi .^{25}$ In all the versions we consider, if one or more $\lambda_{j}, j \neq \Pi$ is allowed to approach infinity, then $\lambda_{\Pi}$ must also be allowed to approach infinity, and the rate of approach of $\lambda_{\Pi}$ must be such that the ratio $\frac{\lambda_{j}}{\lambda_{\Pi}}$ is constant for the $\lambda_{j}$ that is approaching infinity most rapidly. In this version, these restrictions must be met if there is to be a well defined solution for $\ln \Phi$.

Substituting the solutions for $L^{*}$ and $Y^{*}$ into the period utility function (22) with $C_{h}=C=Y$ and $\mathrm{L}_{h}=L$ and rearranging yields ${ }^{26}$

$$
\begin{gathered}
\mathbb{U}^{*}=-K U X^{-\frac{\tilde{\rho} \tilde{\chi}}{J}} Z^{-\frac{\tilde{\alpha} \tilde{\rho}}{J}}, \\
K=\chi_{0}\left(\frac{H}{D}\right)^{\frac{\tilde{\chi}}{J}}\left(\frac{\tilde{\chi} D+\tilde{\alpha} \tilde{\rho}}{\tilde{\alpha} \tilde{\rho} \tilde{\chi}}\right)
\end{gathered}
$$

\footnotetext{
${ }^{25}$ From equation (37) it would appear that it is possible to solve for $\Phi$ as long as $\lambda_{\Pi} \neq 1$. However, in Henderson and Kim (2001) we show that $\lambda_{\Pi}$ must satisfy more stringent conditions in order for there to be a determinate rational expectations equilibrium and that if $\lambda_{\Pi}$ is positive, as seems reasonable and as most if not all investigators have assumed, it must exceed one.

${ }^{26}$ We describe one way to derive equation (38). Use the price equation (price) to eliminate $\frac{\Omega}{\Pi}$ from the wage equation (wage). Note that the resulting equation and the production function (production) imply that $Y^{-\tilde{\rho}} U=\frac{D}{\tilde{\alpha}} \frac{\chi_{0} L^{\tilde{\chi}} U}{Z}$ and use this relationship to eliminate $Y^{-\tilde{\rho}} U$. Then factor out $\frac{\chi_{0} L^{\tilde{\chi}} U}{Z}$ and make use of the solution for $L^{*}$.
} 
Under our assumption that $D>1$, the sign of $K$ is the same as the sign of $\tilde{\rho}$

$$
\operatorname{sgn} K=\operatorname{sgn} \tilde{\rho}
$$

It should come as no surprise that under this assumption utility decreases with increases in the distortion index:

$$
\frac{\partial \mathbb{U}^{*}}{\partial D}=-\left(\frac{\chi_{0}}{J}\right)\left(\frac{H}{D}\right)^{\tilde{\mathcal{J}}}\left(1-\frac{1}{D}\right)\left(U X^{-\frac{\tilde{\tilde{\alpha}} \tilde{x}}{\tau}} Z^{-\frac{\tilde{\alpha} \tilde{\rho}}{\mathcal{J}}}\right)<0
$$

Taking expectations of equation (38) yields the solution for expected utility of the representative consumer with $W^{F} P^{F}$ :

$$
\mathcal{E} \mathbb{U}^{*}=-K \exp \left[\sigma_{u}^{2}+\left(\frac{\tilde{\rho} \tilde{\chi}}{J}\right)^{2} \sigma_{x}^{2}+\left(\frac{\tilde{\alpha} \tilde{\rho}}{J}\right)^{2} \sigma_{z}^{2}\right]
$$

\subsection{Discussion}

We are now prepared to discuss the effects of the shocks on the variables and utility. As should be expected, with $W^{F} P^{F}$ the real variables that enter utility (employment, $L$, and output, $Y$ ) are independent of the parameters of the monetary rule. $L$ and $Y$ depend only on the productivity shock, $X$, and the labor supply shock, $Z$. Expected utility depends on $\sigma_{u}^{2}$ only because $U$ enters the utility function directly.

The effects of a labor supply shock are easier to analyze than those of a productivity shock. The downward sloping marginal product of labor schedule, $M P L$, and the upward sloping marginal rate of substitution (of consumption for labor) schedule, $M R S$, implied by the price equation and wage equation (with $Y$ eliminated using the production function), respectively, are shown in the top panel of Figure 1 in logarithm of real wage $(\omega-\pi)$ and logarithm of employment $(l)$ space. An increase in $Z$ shifts the $M R S$ schedule down from $M R S_{0}$ to $M R S_{1}$. The equilibrium real wage must fall and equilibrium $l$ must rise from $l_{0}$ to $l_{1}$. The upward sloping production function schedule $P F$ is plotted in the bottom panel of Figure 1 in logarithm of output $(y)$ and logarithm of employment space. The increase in $Z$ does not affect the production function, so $y$ rises from $y_{0}$ to $y_{1}$ as $l$ rises from $l_{0}$ to $l_{1}$. An increase in $Z$ raises utility because it results in both an increase in the utility from consumption and a net reduction in the absolute value of the disutility of labor.

Under our assumptions, an increase in productivity (a rise in $X$ ) increases $y$ and lowers, leaves unchanged, or raises $l$ depending on whether $\rho \gtreqless 1$. An increase in $X$ shifts both the $M P L$ and $M R S$ schedules up from $M P L_{0}$ to $M P L_{2}$ and from $M R S_{0}$ to $M R S_{2}$, respectively. The $M R S$ schedule shifts up by more than as in Figure 1, the same as, or less than the MPL schedule depending on whether $\rho \gtreqless 1$. Therefore, the equilibrium real wage must rise and equilibrium $l$ must fall as in Figure 1, remain the same, or rise depending on whether $\rho \gtreqless 1$. An increase in $X$ also shifts the production function to the left from $P F_{0}$ to $P F_{2}$. Equilibrium $y$ definitely rises. Of course, if $l$ rises, $y$ must rise. However, even if $l$ falls as in Figure 1, $y$ rises. $l$ falls only if the $M R S$ schedule shifts up, and the $M R S$ schedule shifts up only if $y$ rises. 
An increase in $X$ raises utility because the increase in the utility from consumption more than offsets any increase in the absolute value of the disutility of labor.

With $W^{F} P^{F}$, the model is recursive. The real variables (labor, output, and the real wage) are determined by the subsystem made up of the production, price, and wage equations. Given values of these variable, the nominal variables (the inflation rate, the nominal interest rate, and the money supply) are determined by the subsystem made up of the demand, rule, and money equations. In our model the only role played by the money market equilibrium condition is to determine the nominal money supply recursively, so we will not make use of it again.

The goods demand shock, $U$, does not enter the subsystem that determines the real variables. An increase in $U$ affects the utility of consumption and the disutility of labor in exactly the same way, so households have no incentive to change their decisions. $U$ enters the subsystem that determines the nominal variables through the policy rule.

\section{Wage Contracts and Flexible Prices}

In this section, we consider the version with wage contracts and flexible prices denoted by $W^{C} P^{F}$ where the superscript $C$ stands for the word 'contracts.'

\subsection{Solution}

In this version, the price and wage equations are

$$
\begin{gathered}
\Pi=\frac{L^{\alpha} \Omega}{X}\left(\frac{\theta_{P}}{s_{P}}\right) \\
\left(\frac{\theta_{W}}{s_{W}}\right) \frac{1}{\Omega}\left[\mathcal{E}_{-1}\left(\frac{\chi_{0} L^{\tilde{\chi}} U}{Z}\right)\right]=\mathcal{E}_{-1}\left(\frac{L U}{Y^{\rho} \Pi}\right)
\end{gathered}
$$

and $\Omega=\frac{W}{P_{-1}}$ is the ratio of the nominal wage to the price level in the previous period. The price equation is the same as in the case of $W^{F} P^{F}$, but the wage equation is different. With wage contracts, the wage and, therefore, $\Omega$ must be set one period in advance without knowledge of the current shocks, so the wage equation contains expectations.

As before, we solve the model using the method of undetermined coefficients. The solutions for selected variables are displayed in Table 2. The solutions for the other variables can be obtained using these solutions and the equations of the model.

Suppose that the solution for $L$ takes the form

$$
L=\Xi U^{\xi_{U}} X^{\xi_{X}} Z^{\xi_{Z}}
$$

We find $\Xi$ by substituting the output and price equations into the wage equation and collecting terms to obtain

$$
\chi_{0} D \mathcal{E}_{-1}\left(\frac{L^{\tilde{\chi}} U}{Z}\right)=\tilde{\alpha}^{\rho} \mathcal{E}_{-1}\left(\frac{U}{L^{\tilde{\alpha} \tilde{\rho}} X^{\tilde{\rho}}}\right) .
$$


Substituting the conjectured form of the solution for $L$ into equation (42) yields

$$
\begin{gathered}
\chi_{0} D \Xi^{\tilde{\chi}} \mathcal{E}_{-1} Q_{3}=\tilde{\alpha}^{\rho} \Xi^{-\tilde{\alpha} \tilde{\rho}} \mathcal{E}_{-1} Q_{2}, \\
Q_{2}=U^{1-\xi_{U} \tilde{\alpha} \tilde{\rho}} X^{-\left(\xi_{X} \tilde{\alpha}+1\right) \tilde{\rho}} Z^{-\xi_{Z} \tilde{\alpha} \tilde{\rho}}, \quad Q_{3}=U^{\xi_{U} \tilde{\chi}+1} X^{\xi_{X}} \tilde{\chi} Z^{\xi_{Z} \tilde{\chi}-1}
\end{gathered}
$$

Therefore, if equation (44) is to hold, $\Xi$ must take on the value in equation $(T 2.3)$ in Table 2 in the Appendix.

We can find the $\xi_{j}$ and $\Omega$ by substituting the rule equation into the demand equation and collecting terms to obtain

$$
U Y^{-\rho}=\Pi^{\lambda_{\Pi}} \Gamma^{\lambda_{\Gamma}} \Upsilon^{\lambda_{\Upsilon}} \Lambda^{\lambda_{\Lambda}} U^{\lambda_{U}} X^{\lambda_{X}} Z^{\lambda_{Z}} \mathcal{E}\left(U_{+1} Y_{+1}^{-\rho} \Pi_{+1}^{-1}\right)
$$

Eliminating $Y, \Pi$, and $Y^{*}$ using the output and price equations and the solution for $Y^{*}$ in equation (35), respectively, collecting some terms, and rearranging yields

$$
\begin{aligned}
& \left(\frac{L^{\tilde{\alpha}} X}{\tilde{\alpha}}\right)^{-\left(\rho+\lambda_{\Gamma}+\lambda_{\Upsilon}\right)} L^{-\lambda_{\Lambda}}\left(\frac{L^{\alpha} \Omega}{X} \frac{\theta_{P}}{s_{P}}\right)^{-\lambda_{\Pi}}\left(\left(\frac{H}{D} X^{-\tilde{\rho}} Z\right)^{\frac{\tilde{\alpha}}{J}} \frac{X}{\tilde{\alpha}}\right)^{\lambda_{\Gamma}} \\
& =Y_{-1}^{-\lambda_{\Upsilon}} L_{-1}^{-\lambda_{\Lambda}} U^{\lambda_{U}-1} X^{\lambda_{X}} Z^{\lambda_{Z}} \mathcal{E}\left(U_{+1}\left(\frac{L_{+1}^{\tilde{\alpha}} X_{+1}}{\tilde{\alpha}}\right)^{-\rho}\left(\frac{L_{+1}^{\alpha} \Omega_{+1}}{X_{+1}} \frac{\theta_{P}}{s_{P}}\right)^{-1}\right)
\end{aligned}
$$

In any period, the variable $\Omega$ is predetermined; that is, $\mathcal{E}\left(\Omega_{+1}\right)=\Omega_{+1}$. This condition

is met by the conjecture $\Omega=Y_{-1}^{\xi_{Y}} L_{-1}^{\xi_{L}} \bar{\Omega}$, where $\bar{\Omega}$ is a constant. This conjecture makes it possible to construct a rational expectations equilibrium. Imposing this conjecture, substituting in the conjectured form for $L$, rearranging, and taking logs yield

$$
\begin{gathered}
\Theta\left(\xi_{U} u+\xi_{X} x+\xi_{Z} z\right)+\lambda_{\Pi} \xi_{Y} y_{-1}+\lambda_{\Pi} \xi_{L} l_{-1}+(\Theta-\rho \tilde{\alpha}-\alpha) \ln \Xi \\
=\left(1-\lambda_{U}\right) u-\left(\frac{\kappa J-\tilde{\chi} \lambda_{\Gamma}}{J}\right) x-\left(\frac{\lambda_{Z} J-\tilde{\alpha} \lambda_{\Gamma}}{J}\right) z+\lambda_{\Upsilon} y_{-1}+\lambda_{\Lambda} l_{-1} \\
-\ln \mathcal{E} Q_{4}+\left(\lambda_{\Upsilon}-\xi_{Y}\right) \ln \tilde{\alpha}-\left(\lambda_{\Pi}-1\right)\left(\bar{\omega}-\ln \left(\frac{s_{P}}{\theta_{P}}\right)\right)+\frac{\lambda_{\Gamma} \tilde{\alpha}}{J} \ln \left(\frac{H}{D}\right) \\
H=\frac{\tilde{\alpha}^{\rho}}{\chi_{0}}, D=\frac{\theta_{W} \theta_{P}}{s_{W} s_{P}}, J=\tilde{\alpha} \tilde{\rho}+\tilde{\chi}, \Theta=\tilde{\alpha}\left(\rho+\lambda_{\Gamma}+\lambda_{\Upsilon}-\xi_{Y}\right)+\left(\lambda_{\Lambda}-\xi_{L}\right)+\alpha \lambda_{\Pi}, \\
\kappa=\lambda_{X}+\rho+\lambda_{\Gamma}+\lambda_{\Upsilon}-\xi_{Y}-\lambda_{\Pi}, \quad Q_{4}=U^{1-\xi_{U}(\tilde{\alpha} \rho+\alpha)} X^{-\left(\tilde{\rho}+\xi_{X}(\tilde{\alpha} \rho+\alpha)\right)} Z^{-\xi_{Z}(\tilde{\alpha} \rho+\alpha)}
\end{gathered}
$$

If equation (47) is to hold for all $U, X$, and $Z$, then the $\xi_{j}$ and $\Omega$ must take on the values given in equations (T2.2) and (T2.8) in Table 2 in the Appendix, respectively. The restrictions on the behavior of $\lambda_{\Pi}$ when one or more $\lambda_{j}, j \neq \Pi$ is allowed to approach infinity stated in the section on the $W^{F} P^{F}$ version must be met if there is to be a well-defined solution for $\bar{\omega}$.

\subsection{The Policymaker's Loss Function}

The policymaker's expected utility function is the expected utility function of the representative agent. However, it simplifies the analysis to define a policymaker's loss function that is a transformation of relative expected utilities. 
The policymaker's expected utility with wage contracts is given by

$$
\mathcal{E} U=\mathcal{E} \mathbb{U}^{*} \exp \left\{\tilde{\alpha} \tilde{\rho} \tilde{\chi}\left[\xi_{U}^{2} \sigma_{u}^{2}+\left(\xi_{X}+\frac{\tilde{\rho}}{J}\right)^{2} \sigma_{x}^{2}+\left(\xi_{Z}-\frac{1}{J}\right)^{2} \sigma_{z}^{2}\right]\right\}
$$

where $\mathcal{E} \mathbb{U}^{*}$ is the policymaker's expected utility with $W^{F} P^{F}$ given in equation (41) which and is invariant to monetary policy. The derivation of this exact expression is actually simpler than the derivation of the standard approximation. ${ }^{27}$

It is helpful to use the concept of certainty equivalent consumption. The utility of consumption, $\mathbb{C}$, is

$$
\mathbb{C}=\frac{C^{1-\rho}}{1-\rho}
$$

and its inverse taking expected utility as an argument is defined as certainty equivalent consumption, $C_{C E}:^{28}$

$$
C_{C E}=[(1-\rho) \mathcal{E} \mathbb{U}]^{\frac{1}{1-\rho}}=[(1-\rho) \mathcal{E} \mathbb{U}]^{-\frac{1}{\tilde{\rho}}}
$$

We define the policymaker's loss function, $\mathbb{L}$, as

$$
\mathbb{L}=\left(\frac{C_{C E}^{*}}{C_{C E}}\right)^{\frac{1}{\tilde{a} \tilde{\chi}}}=\left(\frac{(1-\rho) \mathcal{E} \mathbb{U}}{(1-\rho) \mathcal{E} \mathbb{U}^{*}}\right)^{\frac{1}{\tilde{\alpha} \tilde{\tilde{\chi}} \tilde{\chi}}}=\left(\frac{\mathcal{E} \mathbb{U}}{\mathcal{E} \mathbb{U}^{*}}\right)^{\frac{1}{\tilde{\alpha} \tilde{\rho} \tilde{\chi}}}
$$

It is more convenient to work with the logarithm of loss (hereafter, log loss):

$$
\ln \mathbb{L}=\frac{1}{\tilde{\alpha} \tilde{\chi}}\left(\ln C_{C E}^{*}-\ln C_{C E}\right)=\xi_{U}^{2} \sigma_{u}^{2}+\left(\xi_{X}+\frac{\tilde{\rho}}{J}\right)^{2} \sigma_{x}^{2}+\left(\xi_{Z}-\frac{1}{J}\right)^{2} \sigma_{z}^{2} \geqq 0
$$

From (52) it is apparent that certainty equivalent consumption with $W^{F} P^{F}$ is always greater than or equal to certainty equivalent consumption with $W^{C} P^{F}$. In other words, the best the policymaker can do with wage contracts and flexible prices is to mimic the outcome with full flexibility.

The result just obtained may be somewhat surprising given the distortions arising from monopolistic competition in the product and labor markets, so we consider it in more detail. Expected welfare with $W^{C} P^{F}$ can be written in terms of the mean of the logarithm of labor $(\mathcal{E} \ln L)$ and the variance of a term involving that logarithm and the logarithms of shocks. Furthermore, the policymaker can raise $\mathcal{E} \ln L$ with $W^{C} P^{F}$ above the $W^{F} P^{F}$ level because $\mathcal{E} \ln L$ depends on the variances of the shocks

\footnotetext{
${ }^{27}$ We describe one way to derive $\mathcal{E} U$. In equilibrium $C=Y$. Use the price equation (price) to eliminate $\frac{\Omega}{\Pi}$ from the wage equation (wage). Note that the resulting equation and the production function (production) imply that $\mathcal{E}\left(Y^{-\tilde{\rho}} U\right)=\frac{D}{\tilde{\alpha}} \mathcal{E}\left(\frac{\chi_{0} L^{\tilde{\chi}} U}{Z}\right)$. Use this relationship to eliminate $\mathcal{E}\left(Y^{-\tilde{\rho}} U\right)$ in the expectation of the period utility function $(22)$. Factor out $\mathcal{E}\left(\frac{\chi_{0} L^{\tilde{x}} U}{Z}\right)$ and make use of the solution for $L$ in equation (T2.1) in Table 2 in the Appendix. Make use of the expression for $\mathcal{E} \mathbb{U}^{*}$ in equation (41).

${ }^{28} \mathrm{It}$ is easier to work with $C_{C E}$ than with $\mathcal{E} \mathbb{U}$ because the former is always positive.
} 
and the parameters of the monetary policy rule. However, equation (52) implies that the policymaker has no incentive to raise $\mathcal{E} \ln L$ above the $W^{F} P^{F}$ level because the benefits of doing so are just offset by the costs associated with the required changes in the monetary policy parameters. ${ }^{29}$

\subsection{Full Information}

First we consider the case in which the policymaker can discover the values of all three shocks: the demand shock $U$, the productivity shock $X$, and the labor supply shock $Z$. It can use some combination of inference based on observed values of variables and surveying households and firms. The variables in this version of the model are inflation, output growth, and employment growth. As we will confirm below, the only way for the policymaker to discover the value of the labor supply shock $Z$ is by surveying households because this shock does not affect the values of the variables.

\subsubsection{Optimal Policy}

With wage contracts, the solutions for all the variables depend on the parameters of the monetary rule. Now we derive the optimal full information rule with wage contracts and describe the effects of the shocks under that rule.

In our model, the policymaker must observe and respond appropriately to inflation (or nominal income growth) if there is to be a determinate rational expectations

${ }^{29}$ The discussion in footnote 27 implies that expected utility can be written as

$$
\mathcal{E} \mathbb{U}=\chi_{0}\left(\frac{\tilde{\alpha} \tilde{\rho}+D \tilde{\chi}}{\tilde{\alpha} \tilde{\rho} \tilde{\chi}}\right) \mathcal{E}\left(\frac{L^{\tilde{\chi}} U}{Z}\right)
$$

where

$$
\mathcal{E}\left(\frac{L^{\tilde{\chi}} U}{Z}\right)=\exp \left[\mathcal{E}(\tilde{\chi} \ln L+u-z)+\frac{\mathcal{V}(\tilde{\chi} \ln L+u-z)}{2}\right]=\exp \tilde{\chi}\left[\mathcal{E} \ln L+\frac{\mathcal{V}(\tilde{\chi} \ln L+u-z)}{2}\right]
$$

From the solution for $L$ in the Appendix it follows that

$$
\mathcal{E} \ln L=\frac{\ln \left(\frac{H}{D}\right)}{J}+\left(\xi_{U}^{2} A-2 \xi_{U}\right) \sigma_{u}^{2}+\left(\frac{\xi_{X}^{2} J A+\left(2 \xi_{X} \tilde{\alpha}+1\right) \tilde{\rho}^{2}}{J}\right) \sigma_{x}^{2}+\left(\frac{\xi_{Z}^{2} J A+2 \xi_{Z} \tilde{\chi}-1}{J}\right) \sigma_{z}^{2}
$$

It is true that, for example,

$$
\frac{\partial \mathcal{E} \ln L}{\partial \xi_{U}}=\left(2 \xi_{U} A-2\right) \sigma_{u}^{2}
$$

is negative at $\xi_{U}=0$, the value that makes it possible to replicate the $W^{F} P^{F}$ equilibrium. Therefore, it might seem that expected welfare could be improved by lowering $\xi_{U}$ below zero by raising $\lambda_{U}$ above one. However, the first equation in this footnote can be shown to be equivalent to equation (48) by evaluating $\mathcal{V}(\tilde{\chi} \ln L+u-z)$ and collecting terms, and it is apparent from inspection that the derivative of equation (48) with respect to $\xi_{U}$ is zero at $\xi_{U}=0$. An analogous argument can be made regarding deviations of $\xi_{X}$ and $\xi_{Z}$ from the values that make it possible to replicate the $W^{F} P^{F}$ equilibrium. 
equilibrium. If, in addition, the policymaker can discover the values of all three shocks, it can respond to them or to the output gap and attain the $W^{F} P^{F}$ solution. If the policymaker can respond directly to the shocks or to the output gap,

$$
\lambda_{U}, \lambda_{X}, \lambda_{Z}, \lambda_{\Gamma} \gtreqless 0, \quad \lambda_{\Pi}>1, \quad \lambda_{\Upsilon}=\lambda_{\Lambda}=0
$$

where symbols with prime marks indicate full information values and $\lambda_{\Pi}$ is an arbitrary positive number greater than one.

The solutions for the variables are

$$
\begin{gathered}
\Lambda=\left(\frac{1}{L_{-1}}\right) \Xi U^{\xi_{U}} X^{\xi_{X}} Z^{\xi_{Z}} \\
\Upsilon=\left(\frac{1}{Y_{-1}}\right)\left(\frac{1}{\tilde{\alpha}}\right) \Xi^{\tilde{\alpha}} U^{\xi_{U}}{ }^{\tilde{\alpha}} X^{\xi_{X} \tilde{\alpha}+1} Z^{\xi_{Z} \tilde{\alpha}} \\
\Pi=\left(\frac{\theta_{P}}{s_{P}}\right) \Omega \Xi^{\alpha} U^{\xi_{U} \alpha} X^{\xi_{X} \alpha-1} Z^{\xi_{Z} \alpha} \\
\Xi=\left\{\frac{H}{D}\left[\left(\xi_{U}^{2} A-2 \xi_{U}\right) \sigma_{u}^{2}+\left(\frac{\xi_{X}^{2} J A+\left(2 \xi_{X} \tilde{\alpha}+1\right) \tilde{\rho}^{2}}{J}\right) \sigma_{x}^{2}+\left(\frac{\xi_{Z}^{2} J A+2 \xi_{Z} \tilde{\chi}-1}{J}\right) \sigma_{z}^{2}\right]\right\}^{\frac{1}{J}} \\
\xi_{U}=\frac{1-\lambda_{U}}{\Theta}, \quad \xi_{X}=-\frac{\kappa}{\Theta}+\frac{\tilde{\chi} \lambda_{\Gamma}}{\Theta J}, \quad \xi_{Z}=-\frac{\lambda_{Z}}{\Theta}+\frac{\tilde{\alpha} \lambda_{\Gamma}}{\Theta J}, \quad A=\tilde{\alpha} \tilde{\rho}-\tilde{\chi} \\
\kappa=\lambda_{X}+\rho+\lambda_{\Gamma}-\lambda_{\Pi}, \quad \Theta=\tilde{\alpha}\left(\rho+\lambda_{\Gamma}\right)+\alpha \lambda_{\Pi}, \quad J=\tilde{\alpha} \tilde{\rho}+\tilde{\chi} \\
\Omega=Y_{-1}^{\xi_{Y}} L_{-1}^{\xi_{L}} \bar{\Omega}, \quad \xi_{Y}=\frac{\lambda_{\Upsilon}}{\lambda_{\Pi}}, \xi_{L}=\frac{\lambda_{\Lambda}}{\lambda_{\Pi}}, \bar{\Omega}=\left(\frac{s_{P}}{\theta_{P}}\right)\left(\Xi^{\Theta-\rho \tilde{\alpha}-\alpha}\left(\frac{H}{D}\right)^{-\frac{\lambda_{\Gamma} \tilde{\alpha}}{J}} \mathcal{E} Q_{4}\right)^{-\frac{1}{\lambda_{\Pi}^{-1}}} \\
\ln \mathcal{E} Q_{4}=\left(\left(\frac{1}{\tilde{\alpha} \rho+\alpha}-\xi_{U}\right)^{2} \sigma_{u}^{2}+\left(\xi_{X}+\frac{\tilde{\rho}}{\tilde{\alpha} \rho+\alpha}\right)^{2} \sigma_{x}^{2}+\xi_{Z}^{2} \sigma_{z}^{2}\right)(\tilde{\alpha} \rho+\alpha)^{2}
\end{gathered}
$$

where symbols representing full information values are obtained by beginning with the corresponding symbols in Table 2 in the Appendix and setting $\lambda_{\Upsilon}=\lambda_{\Lambda}=0$. We present solutions for the growth rates of employment and output because we want to consider nominal income growth targeting, and it is convenient to have both real variables expressed in the same terms.

\subsubsection{Offsetting Shocks}

Note that there is a one to one mapping from the parameters of the policy rule to the coefficients of the shocks in the solution for $L$. It is more convenient to determine the optimal shock coefficients for $L$ and then infer the optimal policy rule parameters. It is clear from inspection that the values of the shock coefficients in the solution for labor which minimize (52) are

$$
\xi_{U}=0, \quad \xi_{X}=-\frac{\tilde{\rho}}{J}, \quad \xi_{Z}=\frac{1}{J}
$$


and that the minimum loss is zero. Therefore, if the shock coefficients take on these values expected utility with $W^{C} P^{F}$ is equal to the $W^{F} P^{F}$ level of expected utility.

The optimal rule coefficients implied by the optimal labor coefficients are obtained by equating the expressions for the shock coefficients in equation (T2.2) to the optimal values of these coefficients given in equation (55) and solving for the policy rule parameters. The results are

$$
\lambda_{U}=1, \quad \lambda_{X}=-\frac{\rho(\chi+1)}{\tilde{\alpha} \tilde{\rho}+\tilde{\chi}}+\left(\frac{\rho+\chi}{\tilde{\alpha} \tilde{\rho}+\tilde{\chi}}\right) \lambda_{\Pi}, \quad \lambda_{Z}=-\frac{\tilde{\alpha} \rho}{\tilde{\alpha} \tilde{\rho}+\tilde{\chi}}-\left(\frac{\alpha}{\tilde{\alpha} \tilde{\rho}+\tilde{\chi}}\right) \lambda_{\Pi}
$$

The model exhibits determinacy so long as $\lambda_{\Pi}$ is greater than one, and subject to this constraint the value $\lambda_{\Pi}$ can be chosen arbitrarily. Once a value of $\lambda_{\Pi}$ is chosen, the values of the other policy rule parameters are determined. The policymaker should move the interest rate to exactly match any movement in $U$. It should lower the interest rate if $Z$ rises no matter what the positive value of $\lambda_{\Pi}$ because the marginal disutility of labor varies inversely with $Z$, so output growth and employment growth should be increased. Under some circumstances the policymaker should 'accommodate' the productivity shock by lowering the interest rate. ${ }^{30}$ If $\chi-\lambda_{\Pi}+1>0$ and $\rho$ is large enough that $-\rho\left(\chi-\lambda_{\Pi}+1\right)+\chi \lambda_{\Pi}<0$, then the policymaker should lower the interest rate, but otherwise it should raise the interest rate.

An alternative way of finding the optimal rule is less direct but more elegant. If wages and prices are perfectly flexible and the policymaker follows the optimal rule for which the coefficients are given in equation (56), then for all shocks the utility of the representative consumer is at its second best optimum, and the wage is unaffected. The wage result can be confirmed by substituting the expressions for the $\lambda_{i}$ in equation (56) into the solution for $\Omega^{*}$ in equation (T1.2) in Table 1 in the Appendix. The wage result implies that when the policymaker follows the optimal rule, the outcomes for all the variables including wages are the same no matter whether wages are preset in contracts. That is, the requirement that wages must remain constant is not a constraint that prevents attainment of the second best optimum. It follows that an alternative way of finding the optimal rule with $W^{C} P^{F}$ without ever calculating the solution for that version is to find the rule that keeps wages constant with $W^{F} P^{F}$.

\subsubsection{Strict Output Gap Targeting}

If the policymaker can discover all the shocks, another possible optimal policy is strict output gap targeting. The values of the shocks are used to calculate $W^{F} P^{F}$ output and, therefore, the output gap. The policymaker varies the interest rate so as to totally eliminate deviations of the output gap from zero.

If the policymaker targets a combination of the output gap and inflation so that

$$
\lambda_{\Gamma} \gtreqless 0, \quad \lambda_{\Pi}>1, \quad \lambda_{\Upsilon}=\lambda_{\Lambda}=\lambda_{U}=\lambda_{X}=\lambda_{Z}=0
$$

\footnotetext{
${ }^{30}$ Ireland (1996) finds that with one-period price contracts the policymaker should always accommodate a productivity shock when the money supply is the policy instrument. We obtain an analogous result when the interest rate is the policy instrument in subsection 5.2.
} 
then the values of the shock coefficients in the solution for labor are

$$
\xi_{U}=\frac{1}{\Theta}, \quad \xi_{X}=-\frac{\rho-\lambda_{\Pi}+\lambda_{\Gamma}}{\Theta}+\frac{\tilde{\chi} \lambda_{\Gamma}}{\Theta J}, \quad \xi_{Z}=\frac{\tilde{\alpha} \lambda_{\Gamma}}{\Theta J}
$$

Recall that the model exhibits determinacy if $\lambda_{\Pi}>1$.

Clearly if there is strict output gap targeting so that $\lambda_{\Gamma} \rightarrow \infty$, the values of the shock coefficients in the solution for labor are the $W^{F} P^{F}$ equilibrium values given in equation (55). That is, strict output gap targeting yields the same result as the policy of fully offsetting the shocks discussed in the preceding subsection. This result makes sense because loss can be written as a function of employment and the shocks and by assumption the policymaker knows the shocks and, therefore, can calculate the flexible-price value of employment.

\subsection{Partial Information}

Now we consider the case in which the policymaker can discover information about the shocks only by using observed values of the variables. We explore alternative assumptions about which variables it can observe. In our model, the policymaker must always observe and respond to inflation (or nominal income growth) if there is to be a determinate rational expectations equilibrium. The question then becomes what happens when, in addition, it can observe and respond to output growth or employment growth or both.

If the policymaker cannot directly observe the three shocks, it cannot respond to them or to the output gap which depends on $W^{F} P^{F}$ output and, therefore, on all the shocks. Therefore, ${ }^{31}$

$$
\lambda_{\Upsilon}, \lambda_{\Lambda} \gtreqless 0, \quad \lambda_{\Pi}>1, \quad \lambda_{U}=\lambda_{X}=\lambda_{Z}=\lambda_{\Gamma}=0
$$

The solutions for the variables are given by

$$
\begin{aligned}
& \Lambda=\left(\frac{1}{L_{-1}}\right) \Xi U^{\xi_{U}} X^{\xi_{X}} \\
& \Upsilon=\left(\frac{1}{Y_{-1}}\right)\left(\frac{\Xi^{\tilde{\alpha}}}{\tilde{\alpha}}\right) U^{\xi_{U} \tilde{\alpha}} X^{\xi_{X} \tilde{\alpha}+1} \\
& \Pi=\left(\frac{\theta_{P}}{s_{P}}\right) \Omega \Xi^{\alpha} \frac{L^{\alpha}}{X} U^{\xi_{U} \alpha} X^{\xi_{X} \alpha-1}
\end{aligned}
$$

\footnotetext{
${ }^{31}$ Of course, it could respond to an estimate of the output gap in which estimates of both output and full flexibility output are used. We do not consider this case here. As long as the policymaker is making optimal use of any information it has, it could not do better by responding to an estimate of the output gap.
} 


$$
\begin{aligned}
& \Xi=\left\{\frac{H}{D}\left[\left(\frac{\tilde{\rho}^{2}}{J}\right) \sigma_{x}^{2}-\left(\frac{1}{J}\right) \sigma_{z}^{2}\right]\right\}^{\frac{1}{J}}, \quad \xi_{U}=\frac{1}{\Theta}, \quad \xi_{X}=-\frac{\kappa}{\Theta}, \quad J=\tilde{\alpha} \tilde{\rho}+\tilde{\chi} \\
& \kappa=\rho+\lambda_{\Upsilon}-\xi_{Y}-\lambda_{\Pi}, \Theta=\tilde{\alpha}\left(\rho+\lambda_{\Upsilon}-\xi_{Y}\right)+\left(\lambda_{\Lambda}-\xi_{L}\right)+\alpha \lambda_{\Pi} \\
& \Omega=Y_{-1}^{\xi_{Y}} L_{-1}^{\xi_{L}} \bar{\Omega}, \quad \xi_{Y}=\frac{\lambda_{\Upsilon}}{\lambda_{\Pi}}, \xi_{L}=\frac{\lambda_{\Lambda}}{\lambda_{\Pi}}, \bar{\Omega}=\left(\frac{s_{P}}{\theta_{P}}\right)\left(\Xi^{\Theta-\rho \tilde{\alpha}-\alpha} \tilde{\alpha}^{-\left(\lambda_{\Upsilon}-\xi_{Y}\right)} \mathcal{E} Q_{4}\right)^{-\frac{1}{\lambda_{\Pi}-1}} \\
& \ln \mathcal{E} Q_{4}=\sigma_{u}^{2}+\tilde{\rho}^{2} \sigma_{x}^{2}+(\tilde{\alpha} \rho+\alpha)^{2} \sigma_{z}^{2}
\end{aligned}
$$

where symbols representing partial information are obtained from the corresponding symbols in Table 2 in the Appendix by setting $\lambda_{U}=\lambda_{X}=\lambda_{Z}=\lambda_{\Gamma}=0$.

One might conjecture that if the policymaker could observe and respond to output growth and employment growth as well as to inflation, then it could achieve the $W^{F} P^{F}$ equilibrium. After all, in that case there would be three information variables, and there are only three shocks. However, it is clear from equations (60), that this conjecture is incorrect. The three information variables all depend on only two shocks, the demand shock $U$ and the productivity shock $X$. Since nominal wages are set in one period contracts before markets meet and households agree to supply whatever labor firms want at those wages, the labor supply shock $Z$ has no effect on the equilibrium values of the variables.

What is true is that if the policymaker can observe either output growth or employment growth in addition to inflation, it can determine the values of $U$ and $X$ and can offset the effects of these shocks on loss and can do nothing to offset the effects of $Z$ on loss. We illustrate this general result with the case in which the policymaker can observe and respond to inflation and output growth in the next subsection but we do not work out the other possible case.

If the policymaker can observe and respond only to inflation or to a combination of inflation and some other variable such as nominal income growth, it can determine only a combination of the values of $U$ and $X$. In this situation, the policymaker should choose its response based on the relative importance of the two disturbances. We also illustrate this general result with an example.

As the language of this section suggests, the policymaker's stabilization problem can be viewed as a 'signal extraction' problem, where the 'signals' are movements in observed economic variables and what are to be 'extracted' are estimates of the shocks. $^{32}$ In our exposition, we do not explicitly view the problem in that way but instead view it as a problem of choosing the best possible monetary rule coefficients given the information constraints. Our way of viewing the problem is a little less transparent but considerably simpler computationally.

\footnotetext{
${ }^{32}$ Kalchbrenner, Tinsley, Berry, and Garrett (1977) and LeRoy and Waud (1977) were among the first to emphasize how instructive it is to cast stabilization problems as signal extraction problems.
} 


\subsubsection{Targeting a Combination of Inflation and Output Growth}

Suppose that the policymaker can observe and respond to both inflation and output growth. Then log loss is

$$
\begin{aligned}
\left.\ln \mathbb{L}\right|^{\Pi, \Upsilon}= & \left(\frac{1}{\tilde{\alpha}\left(\rho+\lambda_{\Upsilon}-\frac{\lambda_{\Upsilon}}{\lambda_{\Pi}}\right)+\alpha \lambda_{\Pi}}\right)^{2} \sigma_{u}^{2} \\
& +\left(-\frac{\rho+\lambda_{\Upsilon}-\frac{\lambda_{\Upsilon}}{\lambda_{\Pi}}-\lambda_{\Pi}}{\tilde{\alpha}\left(\rho+\lambda_{\Upsilon}-\frac{\lambda_{\Upsilon}}{\lambda_{\Pi}}\right)+\alpha \lambda_{\Pi}}+\frac{\tilde{\rho}}{J}\right)^{2} \sigma_{x}^{2}+\left(\frac{1}{J}\right)^{2} \sigma_{z}^{2}
\end{aligned}
$$

where the superscript after the vertical bar indicates which variable or set of variables is being observed and responded to.

In accordance with the principle stated in the last subsection, the loss associated with the variances of $U$ and $X$ can be totally eliminated but the loss associated with the variance of $Z$ can not be reduced. ${ }^{33}$ In particular, if ${ }^{34}$

$$
\frac{\lambda_{\Upsilon}}{\lambda_{\Pi}}=\frac{\tilde{\rho}+\tilde{\chi}}{\tilde{\chi}}=\frac{\tilde{\rho}}{\tilde{\chi}}+1>0, \quad \lambda_{\Upsilon} \rightarrow \infty
$$

then $\log \operatorname{loss}$ is

$$
\left.\ln \mathbb{L}\right|_{O} ^{\Pi, \Upsilon}=\left(\frac{1}{J}\right)^{2} \sigma_{z}^{2}
$$

where the subscript $O$ indicates optimal stabilization. Note that the ratio $\frac{\lambda_{r}}{\lambda_{\Pi}}$ is higher than the ratio $\frac{\tilde{\rho}}{\tilde{\chi}}$, that is, there is more response to output growth relative to inflation the more costly is output (consumption) variation relative to employment variation.

There is an alternative way of finding the optimal responses to inflation and output growth which is analogous to the alternative way of finding the fully optimal rule discussed in the subsection on offsetting shocks. The optimal responses to inflation and output growth in the version with $W^{C} P^{F}$ are those that would make the nominal wage invariant to demand and productivity shocks $(U$ and $X)$ in the version with $W^{F} P^{F}$. The solution for the nominal wage with $W^{F} P^{F}$ is given in equation (T1.2) and the nominal wage is invariant to $U$ and $X$ if and only if the $\lambda_{i}$ are set at the values given in equation (62).

\footnotetext{
${ }^{33}$ This result was obtained by Koenig (1996) who allows for separate responses to the levels of price and output.

${ }^{34}$ In order to confirm the result stated in equation (62), divide the numerators and denominators of the coefficients of $\sigma_{u}^{2}$ and $\sigma_{x}^{2}$ by $\lambda_{\Pi}$, let $\lambda_{\Pi}$ and $\lambda_{\Upsilon}$ approach infinity holding the ratio $\frac{\lambda_{\Upsilon}}{\lambda_{\Pi}}$ constant. Taking this limit drives the coefficient on $\sigma_{u}^{2}$ to zero. The coefficient on $\sigma_{x}^{2}$ can be set to zero by choosing the indicated value for $\frac{\lambda_{\Upsilon}}{\lambda_{\Pi}}$.
} 


\subsubsection{Nominal Income Growth}

Now suppose that the policymaker can observe and respond to only nominal income growth. In this case the policymaker is responding to the single variable $\Pi \Upsilon$, so it can infer only a combination of the $U$ and $X$ shocks, not the two shocks individually. Therefore, it cannot fully offset these shocks.

If the nominal interest rate responds only to deviations of nominal income growth from a constant target value of 0 , so that

$$
\lambda_{\Pi}=\lambda_{\Upsilon}>1
$$

then $\log \operatorname{loss}$ is

$$
\left.\ln \mathbb{L}\right|^{\Pi \Upsilon}=\left(\frac{1}{\tilde{\alpha} \tilde{\rho}+\lambda_{\Upsilon}}\right)^{2} \sigma_{u}^{2}+\left(\frac{-\tilde{\rho}}{\tilde{\alpha} \tilde{\rho}+\lambda_{\Upsilon}}+\frac{\tilde{\rho}}{J}\right)^{2} \sigma_{x}^{2}+\left(\frac{1}{J}\right)^{2} \sigma_{z}^{2}
$$

Under strict nominal income growth targeting, $\lambda_{\Pi}=\lambda_{\Upsilon} \rightarrow \infty$, and log loss is

$$
\left.\ln \mathbb{L}\right|_{S} ^{\Pi \Upsilon}=\left(\frac{\tilde{\rho}}{J}\right)^{2} \sigma_{x}^{2}+\left(\frac{1}{J}\right)^{2} \sigma_{z}^{2}
$$

where the subscript $S$ indicates strict targeting or total stabilization. Note that the more inelastic is labor supply (the larger $\chi$ and, therefore, the larger is $J$ ) the closer is strict nominal income growth targeting to the fully optimal policy. ${ }^{35}$

Optimal nominal income growth targeting involves minimizing the log loss in equation (65) with respect to $\lambda_{\Upsilon}$. The optimal $\lambda_{\Upsilon}$ is

$$
\lambda_{\Upsilon}=\frac{J \sigma_{u}^{2}+\tilde{\rho}^{2} \tilde{\chi} \sigma_{x}^{2}}{\tilde{\rho}^{2} \sigma_{x}^{2}}=\frac{J}{\tilde{\rho}^{2}} \frac{\sigma_{u}^{2}}{\sigma_{x}^{2}}+\tilde{\chi}=\frac{J}{\tilde{\rho}^{2}} \frac{\sigma_{u}^{2}}{\sigma_{x}^{2}}+(1+\chi)>1
$$

The optimal interest rate response increases with the ratio of the variance of the demand shock to the variance of the productivity shock. As we confirm below, the property that the optimal response coefficient rises with ratio $\frac{\sigma_{u}^{2}}{\sigma_{x}^{2}}$ is quite general.

The log loss from the optimal response to nominal income growth can be expressed as a positive fraction of the log loss associated with the productivity shock under strict nominal income growth targeting plus the irreducible loss associated with the labor supply shock:

$$
\left.\ln \mathbb{L}\right|_{O} ^{\Pi \Upsilon}=\left(\frac{\sigma_{u}^{2}}{\tilde{\rho}^{2} \sigma_{x}^{2}+\sigma_{u}^{2}}\right)\left(\frac{\tilde{\rho}}{J}\right)^{2} \sigma_{x}^{2}+\left(\frac{1}{J}\right)^{2} \sigma_{z}^{2}
$$

The fraction rises from zero to one as the ratio $\frac{\sigma_{u}^{2}}{\sigma_{x}^{2}}$ increases from zero to infinity. In other words, the larger the ratio of the variance of the demand shock to the variance of the productivity shock, the larger the ratio of the optimal loss to the loss with strict nominal income targeting, that is, the less suboptimal is strict nominal income targeting. As we confirm below, the property that the ratio of optimal loss to the strict-targeting loss rises with the ratio $\frac{\sigma_{u}^{2}}{\sigma_{x}^{2}}$ is also quite general. The explanation for

\footnotetext{
${ }^{35}$ This result was obtained by Bean (1983).
} 
this property is that if the only shocks are demand shocks, then total stabilization of any of $\Pi \Upsilon, \Pi, \Upsilon$, or $\Lambda$ yields the best feasible result. However, if there are also productivity shocks, then, in general, the best feasible result requires changes in all of these variables.

\subsubsection{Inflation}

Next suppose that the policymaker can observe and respond only to deviations of the inflation rate from a constant target value. It should be emphasized that we have already shown that if the policymaker has information on either output growth or employment, it should use that information. Thus, the only reason why a policymaker would respond only to inflation is that information about inflation is the only information that it has.

If the policymaker can observe and respond only to the inflation rate, then

$$
\lambda_{\Pi}>1, \quad \lambda_{\Upsilon}=\lambda_{\Gamma}=\lambda_{U}=\lambda_{X}=\lambda_{Z}=0
$$

then $\log \operatorname{loss}$ is

$$
\begin{gathered}
\left.\ln \mathbb{L}\right|^{\Pi=}\left(\frac{1}{\Theta_{\Pi}}\right)^{2} \sigma_{u}^{2}+\left(\frac{\lambda_{\Pi}-\rho}{\Theta_{\Pi}}+\frac{\tilde{\rho}}{J}\right)^{2} \sigma_{x}^{2}+\left(\frac{1}{J}\right)^{2} \sigma_{z}^{2} \\
\Theta_{\Pi}=\tilde{\alpha} \rho+\alpha \lambda_{\Pi}
\end{gathered}
$$

Under strict inflation targeting, log loss is

$$
\left.\ln \mathbb{L}\right|_{S} ^{\Pi}=\left(\frac{1}{\alpha}+\frac{\tilde{\rho}}{J}\right)^{2} \sigma_{x}^{2}+\left(\frac{1}{J}\right)^{2} \sigma_{z}^{2}=\left(\frac{\rho+\chi}{\alpha J}\right)^{2} \sigma_{x}^{2}+\left(\frac{1}{J}\right)^{2} \sigma_{z}^{2}
$$

For labor supply shocks, employment growth and, therefore, output growth are unaffected, and are, therefore, less volatile than under the optimal policy. For productivity shocks, under strict inflation targeting, output growth is always more volatile than under the optimal policy and employment growth is more volatile than under the optimal policy for a wide range of parameters with $\alpha<\frac{1}{2}$ being a sufficient condition.

Optimal inflation targeting involves minimizing the log loss given by equation (70) with respect to $\lambda_{\Pi}$ which yields

$$
\lambda_{\Pi}=\frac{\rho \tilde{\chi}}{\tilde{\rho}+\tilde{\chi}}+\frac{\alpha J}{\rho(\tilde{\rho}+\tilde{\chi})} \frac{\sigma_{u}^{2}}{\sigma_{x}^{2}}
$$

$\lambda_{\Pi}$ rises with $\frac{\sigma_{u}^{2}}{\sigma_{x}^{2}}$.

Note that $\lambda_{\Pi}^{x}$ must exceed one if the model is to have a unique rational expectations equilibrium. The optimal value of $\lambda_{\Pi}$ exceeds one if and only if

$$
\frac{\rho \tilde{\chi}}{\tilde{\rho}+\tilde{\chi}}+\frac{\alpha J}{\rho(\tilde{\rho}+\tilde{\chi})} \frac{\sigma_{u}^{2}}{\sigma_{x}^{2}}>1 \Leftrightarrow(\rho-1) \rho \chi+\alpha[(1-\alpha) \rho+\alpha+\chi] \frac{\sigma_{u}^{2}}{\sigma_{x}^{2}}>0
$$


This condition is definitely met if $\rho \geq 1$ and may be met if $\rho<1$. If this condition is not met, there is no unique feasible value of $\lambda_{\Pi}$ that minimizes log loss; the smaller the amount by which $\lambda_{\Pi}$ exceeds one, the lower is log loss.

The log loss from the optimal response to inflation is a positive fraction of the log loss associated with the productivity shock under strict inflation targeting plus the irreducible loss associated with the labor supply shock:

$$
\left.\ln \mathbb{L}\right|_{O} ^{\Pi}=\left(\frac{\alpha^{2} \sigma_{u}^{2}}{\rho^{2} \sigma_{x}^{2}+\alpha^{2} \sigma_{u}^{2}}\right)\left(\frac{\rho+\chi}{\alpha J}\right)^{2} \sigma_{x}^{2}+\left(\frac{1}{J}\right)^{2} \sigma_{z}^{2}
$$

The fraction rises from zero to one as the ratio $\frac{\sigma_{u}^{2}}{\sigma_{x}^{2}}$ increases from zero to infinity.

\subsubsection{Comparison}

Nominal income targeting is better than inflation targeting for plausible values of the parameters. A necessary and sufficient condition for strict nominal income targeting to be better than strict inflation targeting is

$$
\left(\frac{\tilde{\rho}}{J}\right)^{2}<\left(\frac{\tilde{\rho}+\tilde{\chi}}{\alpha J}\right)^{2}
$$

If $\rho>1$, this condition is always fulfilled. If $\rho<1$, this condition can be reduced to

$$
\rho>\frac{\alpha-\chi}{\alpha+1}
$$

where the maximum value of the right hand side is $\frac{1}{2}$, attained when $\alpha \rightarrow 1$ and $\chi \rightarrow 0$. The majority of empirical studies find point estimates of $\rho$ that are greater than one, often much greater. The condition in equation (75) is sufficient for optimal nominal income growth targeting to be better than optimal inflation targeting. ${ }^{36}$

What accounts for the general superiority of nominal income growth targeting? The two policies are equally effective in offsetting demand shocks: the strict version of each leads to a complete offsetting of demand shocks. However, nominal income growth targeting is better at offsetting productivity shocks for plausible parameter values. According to equation (62), the optimal separate responses to inflation and output growth have the same sign but are not equal in value except in the special case in which $\rho=1$. Consider the ratio of the income growth response to the inflation response with various rules. A sufficient condition for the ratio of the optimal separate responses to be closer to the ratio of responses with nominal income growth targeting,

\footnotetext{
${ }^{36}$ The necessary and sufficient condition for optimal nominal income growth targeting to be better than optimal inflation targeting reduces to
}

$$
\tilde{\rho}^{2} \rho^{2} \sigma_{x}^{2}+\tilde{\rho}^{2} \alpha^{2} \sigma_{u}^{2}<(\rho+\chi)^{2}\left[\tilde{\rho}^{2} \sigma_{x}^{2}+\sigma_{u}^{2}\right]
$$

Condition (75) implies

$$
\tilde{\rho}^{2} \alpha^{2} \sigma_{u}^{2}<(\rho+\chi)^{2} \sigma_{u}^{2}, \quad \tilde{\rho}^{2} \rho^{2} \sigma_{x}^{2}<\tilde{\rho}^{2}(\rho+\chi)^{2} \sigma_{x}^{2}
$$


which equals unity, than to the ratio with inflation targeting, which equals zero, is $\rho>\frac{1}{2}$.

\section{$5 \quad$ Wage and Price Contracts}

In this section we consider the version with both wage and price contracts denoted by $W^{C} P^{C}$.

\subsection{Solution}

With $W^{C} P^{C}$ the wage equation is the same as with $W^{C} P^{F}$, but the price equation is different from that in both of the other versions:

$$
\begin{gathered}
\mathcal{E}_{-1}\left(\frac{U}{Y^{\tilde{\rho}}}\right)=\frac{\Omega}{\Pi}\left[\mathcal{E}_{-1}\left(\frac{L^{\alpha} U}{Y^{\tilde{\rho}} X}\right)\right]\left(\frac{\theta_{P}}{s_{P}}\right) \\
\left(\frac{\theta_{W}}{s_{W}}\right)\left[\mathcal{E}_{-1}\left(\frac{\chi_{0} L^{\tilde{\chi}} U}{Z}\right)\right]=\frac{\Omega}{\Pi}\left[\mathcal{E}_{-1}\left(\frac{L U}{Y^{\rho}}\right)\right]
\end{gathered}
$$

Not only wages but also prices must be set one period in advance without knowledge of the current shocks so not only the wage equation but also the price equation contain expectations.

We solve the model using the method of undetermined coefficients. The general solutions are displayed in Table 3 in the Appendix. Suppose that the solution for $L$ has the form

$$
L=\Psi U^{\psi_{U}} X^{\psi_{X}} Z^{\psi_{Z}}
$$

We find $\Psi$ by substituting the production equation into the price and wage equations,

collecting terms, and dividing the price equation by the wage equation to eliminate $\frac{\Omega}{\Pi}$ to obtain

$$
\frac{\mathcal{E}_{-1}\left(L^{-\tilde{\alpha} \tilde{\rho}} U X^{-\tilde{\rho}}\right)}{\chi_{0} \mathcal{E}_{-1}\left(L \tilde{\chi} U Z^{-1}\right)}=\frac{D \mathcal{E}_{-1}\left(L^{\alpha-\tilde{\alpha} \tilde{\rho}} U X^{-\rho}\right)}{\tilde{\alpha}^{\rho} \mathcal{E}_{-1}\left(L^{\alpha-\tilde{\alpha} \tilde{\rho}} U X^{-\rho}\right)}
$$

Substituting in the conjectured form of the solution for $L$ in equation (77) and rearranging yields

$$
\begin{gathered}
\frac{\tilde{\alpha}^{\rho} \Psi^{-\tilde{\alpha} \tilde{\rho}} \mathcal{E}_{-1}\left(Q_{5}\right)}{\chi_{0} \Psi \tilde{\chi} \mathcal{E}_{-1}\left(Q_{6}\right)}=D \\
Q_{5}=U^{1-\psi_{U} \tilde{\alpha} \tilde{\rho}} V^{-\psi_{V} \tilde{\alpha} \tilde{\rho}} X^{-\left(\psi_{X} \tilde{\alpha} \tilde{\rho}+\tilde{\rho}\right)} Z^{-\psi_{Z} \tilde{\alpha} \tilde{\rho}}, \quad Q_{6}=U^{\psi_{U} \tilde{\chi}+1} V^{\psi_{V} \tilde{\chi}} X^{\psi_{X} \tilde{\chi}} Z^{\psi_{Z} \tilde{\chi}-1}
\end{gathered}
$$

If equation (79) is to hold $\Psi$ must take on the value in equation (T3.4).

We find the $\psi_{j}, \Pi$, and $\Omega$ by substituting the rule equation into the demand equation to obtain

$$
Y^{-\rho} U=\Pi^{\lambda_{\Pi}} \Gamma^{\lambda_{\Gamma}} \Upsilon^{\lambda_{\Upsilon}} \Lambda^{\lambda_{\Lambda}} U^{\lambda_{U}} X^{\lambda_{X}} Z^{\lambda_{Z}} \mathcal{E}\left(Y_{+1}^{-\rho} \Pi_{+1}^{-1} U_{+1}\right)
$$


In any period, $\Pi$ is predetermined; that is, $\mathcal{E}\left(\Pi_{+1}\right)=\Pi_{+1}$. This condition is met by the conjecture $\Pi=Y_{-1}^{\psi_{Y}} L_{-1}^{\psi_{L}} \bar{\Pi}$, where $\bar{\Pi}$ is a constant. This conjecture makes it possible to construct a rational expectations equilibrium. Imposing this conjecture, substituting for $Y$ the form implied by the conjectured form for $L$, rearranging, and taking logs yield

$$
\begin{aligned}
& \digamma\left(\psi_{U} u+\psi_{X} x+\psi_{Z} z\right)+\lambda_{\Pi} \psi_{Y} y_{-1}+\lambda_{\Pi} \psi_{L} l_{-1}+(\digamma-\rho \tilde{\alpha}) \ln \Psi \\
= & \left(1-\lambda_{U}\right) u-\left(\frac{\varepsilon J-\tilde{\chi} \lambda_{\Gamma}}{J}\right) x-\left(\frac{\lambda_{Z} J-\tilde{\alpha} \lambda_{\Gamma}}{J}\right) z+\lambda_{\Upsilon} y_{-1}+\lambda_{\Lambda} l_{-1} \\
& +\left(\lambda_{\Upsilon}-\psi_{Y}\right) \ln \tilde{\alpha}+\frac{\lambda_{\Gamma} \tilde{\alpha}}{J} \ln \left(\frac{H}{D}\right)-\ln \mathcal{E} Q_{7}-\left(\lambda_{\Pi}-1\right) \bar{\pi} \\
\digamma= & \tilde{\alpha}\left(\rho+\lambda_{\Gamma}+\lambda_{\Upsilon}-\psi_{Y}\right)+\left(\lambda_{\Lambda}-\psi_{L}\right), \quad \varepsilon=\lambda_{X}+\rho+\lambda_{\Gamma}+\lambda_{\Upsilon}-\psi_{Y} \\
J= & \tilde{\alpha} \tilde{\rho}+\tilde{\chi}, \quad Q_{7}=U^{1-\psi_{U} \tilde{\alpha} \rho} X^{-\psi_{X} \tilde{\alpha} \rho-\rho} Z^{-\psi_{Z} \tilde{\alpha} \rho}
\end{aligned}
$$

If equation (81) is to hold for all $U, X$, and $Z$, it must be that the $\psi_{j}$ and $\Pi$, respectively, must take on the values given in equations (T3.2) and (T3.8) in Table 3 in the Appendix. Given the solution for $\Pi$, the price equation can be used to obtain the solution for $\Omega$ in equation (T3.10) in Table $3 .{ }^{37}$ The restrictions on the behavior of $\lambda_{\Pi}$ when one or more $\lambda_{j}, j \neq \Pi$ is allowed to approach infinity stated in the section on the $W^{F} P^{F}$ version must be met if there is to be a well-defined solution for $\bar{\pi}$.

\subsection{Full Information}

In this subsection we derive the optimal monetary policy with $W^{C} P^{C}$ under the assumption that the policymaker has full information about the current values of all the shocks. With $W^{C} P^{C}$, just as with $W^{C} P^{F}$, output gap targeting yields the optimal outcome and for the same reason.

The solutions for the variables are

$$
\begin{gathered}
\Lambda=\left(\frac{1}{L_{-1}}\right) \Psi U^{\psi_{U}} X^{\psi_{X}} Z^{\psi_{Z}} \\
\Upsilon=\left(\frac{1}{Y_{-1}}\right)\left(\frac{1}{\tilde{\alpha}}\right) \Psi^{\tilde{\alpha}} U^{\psi_{U} \tilde{\alpha}} X^{\psi_{X} \tilde{\alpha}+1} Z^{\psi_{Z} \tilde{\alpha}} \\
\psi_{U}=\frac{1-\lambda_{U}}{\digamma}, \psi_{X}=-\frac{\varepsilon}{\digamma}+\frac{\tilde{\chi} \lambda_{\Gamma}}{\digamma J}, \psi_{Z}=-\frac{\lambda_{Z}}{\digamma}+\frac{\tilde{\alpha} \lambda_{\Gamma}}{\digamma J} \\
\varepsilon=\lambda_{X}+\rho+\lambda_{\Gamma}, \quad \digamma=\tilde{\alpha}\left(\rho+\lambda_{\Gamma}\right), \quad J=\tilde{\alpha} \tilde{\rho}+\tilde{\chi}
\end{gathered}
$$

where symbols for full information are obtained by starting with the corresponding symbols in Table 3 and setting $\lambda_{\Upsilon}=\lambda_{\Lambda}=0$.

As in the case of $W^{C} P^{F}$, we state the policymaker's optimization problem in terms of the labor coefficients and then infer the optimal rule coefficients. It is clear from Tables 2 and 3 that the solution for $L$ and, therefore, the solution for $Y$ have exactly

\footnotetext{
${ }^{37}$ Of course, the solution for $\Omega$ can also be obtained using the wage equation.
} 
the same form with $W^{C} P^{C}$ as they do with wage contracts alone with $\psi_{j}, j=U, X, Z$ replacing $\xi_{j}, j=U, X, Z$ wherever they appear. It follows that the expressions for expected loss and, therefore, the optimal values of the shock coefficients in the solution for $L$ are the same with $W^{C} P^{C}$ as they are with wage contracts alone. That is,

$$
\psi_{U}=0, \quad \psi_{X}=-\frac{\tilde{\rho}}{J}, \quad \psi_{Z}=\frac{1}{J}
$$

In characterizing the optimal policy rule, as before we assume that the policymaker responds either to the shocks and inflation or to the output gap and inflation:

$$
\lambda_{U}, \lambda_{X}, \lambda_{Z} \gtreqless 0 \text { or } \lambda_{\Gamma} \gtreqless 0, \quad \lambda_{\Pi}>1, \quad \lambda_{\Upsilon}=\lambda_{\Lambda}=0
$$

and that $\lambda_{\Pi}$ is an arbitrary positive number greater than one. The optimal shock coefficients implied by the optimal labor coefficients are

$$
\lambda_{U}=1, \quad \lambda_{X}=-\frac{\rho \tilde{\chi}}{J}, \quad \lambda_{Z}=-\frac{\tilde{\alpha} \rho}{J}
$$

According to the expression for $\lambda_{X}$ in equation (85), the policymaker should always accommodate productivity shocks. This result is analogous to the finding of Ireland (1996) who assumes that the money supply is the policy instrument.

The optimal output gap coefficient implied by the optimal labor coefficient is

$$
\lambda_{\Gamma} \rightarrow \infty
$$

In contrast to the results for wage contracts alone, with $W^{C} P^{C}$ the optimal $\lambda_{j}, j=$ $U, X, Z$ are independent of $\lambda_{\Pi}$. The only role played by $\lambda_{\Pi}$ is to guarantee determinacy, in particular, to insure that agents can calculate the expected future inflation. The contract price for the current period is set before the shocks are drawn so there can be no movements in current inflation induced by the shocks and therefore nothing for the policymaker to respond to. Recall that $\lambda_{\Pi}$ must be allowed to approach infinity at the same rate as $\lambda_{\Gamma}$ if there is to be a well defined solution for $\bar{\pi}$ in equation (81).

With $W^{C} P^{C}$, just as with $W^{C} P^{F}$, there is an alternative way of finding the optimal rule that is less direct but more elegant. The alternative way of finding the optimal $\lambda_{j}, j=U, X, Z$ in the version with $W^{C} P^{C}$ is to find the values of those coefficients that keep prices constant in the version with $W^{F} P^{F}$. The logic that leads to this result may not be immediately obvious. As we show in Henderson and Kim (1999), outcomes with price contracts and flexible wages $\left(W^{F} P^{C}\right)$ are the same as the outcomes with $W^{C} P^{C}$ for all variables except the nominal wage. Therefore, the optimal rule with $W^{C} P^{C}$ is the same as the optimal rule with $W^{F} P^{C}$. By analogy with case of $W^{C} P^{F}$, the optimal $\lambda_{j}, j=U, X, Z$ in the version with $W^{F} P^{C}$ can be obtained by finding the values of those coefficients that keep prices constant in the version with $W^{F} P^{F}$. 


\subsection{Partial Information}

Now we consider the case in which the policymaker can discover information about the shocks only by using observed values of the variables. We explore alternative assumptions about which variables it can observe. In our model, the policymaker must always observe and respond to inflation (or nominal income growth) if there is to be a determinate rational expectations equilibrium. The question then becomes what happens when, in addition, it can observe and respond to output growth or employment growth or both.

If the policymaker cannot directly observe the three shocks, it cannot respond to them or to the output gap which depends on $W^{F} P^{F}$ output and, therefore, on all the shocks: ${ }^{38}$

$$
\lambda_{\Upsilon}, \lambda_{\Lambda} \gtreqless 0, \quad \lambda_{\Pi}>1, \quad \lambda_{U}=\lambda_{X}=\lambda_{Z}=\lambda_{\Gamma}=0
$$

The solutions for the variables are

$$
\begin{gathered}
\Lambda=\left(\frac{1}{L_{-1}}\right) \Psi U^{\psi_{U}} X^{\psi_{X}} \\
\Upsilon=\left(\frac{1}{Y_{-1}}\right)\left(\frac{1}{\tilde{\alpha}}\right) \Psi^{\tilde{\alpha}} U^{\psi_{U} \tilde{\alpha}} \\
\psi_{U}=\frac{1}{\digamma}, \quad \psi_{X}=-\frac{\varepsilon}{\digamma}, \quad \psi_{Y}=\frac{\lambda_{\Upsilon}}{\lambda_{\Pi}}, \quad \psi_{L}=\frac{\lambda_{\Lambda}}{\lambda_{\Pi}}, \\
\varepsilon=\rho+\lambda_{\Upsilon}-\psi_{Y}, \quad \digamma=\tilde{\alpha}\left(\rho+\lambda_{\Upsilon}-\psi_{Y}\right)+\left(\lambda_{\Lambda}-\psi_{L}\right), \quad J=\tilde{\alpha} \tilde{\rho}+\tilde{\chi}
\end{gathered}
$$

where symbols for partial information are obtained by starting with the corresponding symbols in Table 3 in the Appendix and setting $\lambda_{U}=\lambda_{X}=\lambda_{Z}=\lambda_{\Gamma}=0$.

Suppose the nominal interest rate responds to deviations in both output growth and labor growth from unity, so that

$$
\lambda_{\Upsilon}>0, \quad \lambda_{\Lambda}>0, \quad \lambda_{\Pi}>1, \quad \lambda_{\Gamma}=\lambda_{U}=\lambda_{X}=\lambda_{Z}=0
$$

where symbols for partial information are obtained by starting with the corresponding symbols in Table 3 and setting $\lambda_{U}=\lambda_{X}=\lambda_{Z}=\lambda_{\Gamma}=0$. In this case, log loss is

$$
\begin{aligned}
\left.\ln \mathbb{L}\right|^{\Upsilon, \Lambda}= & \left(\frac{1}{\tilde{\alpha}\left(\rho+\lambda_{\Upsilon}-\psi_{Y}\right)+\left(\lambda_{\Lambda}-\psi_{L}\right)}\right)^{2} \sigma_{u}^{2} \\
& +\left(-\frac{\left(\rho+\lambda_{\Upsilon}-\psi_{Y}\right)}{\tilde{\alpha}\left(\rho+\lambda_{\Upsilon}-\psi_{Y}\right)+\left(\lambda_{\Lambda}-\psi_{L}\right)}+\frac{\tilde{\rho}}{J}\right)^{2} \sigma_{x}^{2}+\left(\frac{1}{J}\right)^{2} \sigma_{z}^{2}
\end{aligned}
$$

If the policymaker observes and responds to both output growth and employment growth, the loss associated with the variances of $U$ and $X$ can be totally eliminated

\footnotetext{
${ }^{38}$ Of course, it could respond to an estimate of the output gap in which estimates of both output and full flexibility output are used. We do not consider this case here. As long as the policymaker is making optimal use of any information it has, it could not do better by responding to an estimate of the output gap.
} 
but the loss associated with the variance of $Z$ can not be reduced. In particular, if ${ }^{39}$

$$
\frac{\lambda_{\Upsilon}}{\lambda_{\Lambda}}=\frac{\tilde{\rho}}{\tilde{\chi}}>0, \quad \lambda_{\Upsilon} \rightarrow \infty, \quad \frac{\lambda_{\Pi}}{\lambda_{\Upsilon}}=\mathrm{constant}, \quad \lambda_{\Gamma}=\lambda_{U}=\lambda_{X}=\lambda_{Z}=0
$$

then $\log \operatorname{loss}$ is

$$
\left.\ln \mathbb{L}\right|_{O} ^{\Upsilon, \Lambda}=\left(\frac{1}{J}\right)^{2} \sigma_{z}^{2}
$$

where the subscript $O$ indicates optimal stabilization. Note that the ratio $\frac{\lambda_{\Upsilon}}{\lambda_{\Lambda}}$ is higher the higher the ratio $\frac{\tilde{\rho}}{\tilde{\chi}}$, that is, there is more response to output growth relative to employment growth the more costly is output (consumption) variation relative to employment variation.

There is an alternative way of finding the optimal responses to output growth and employment growth which is analogous to the alternative ways of finding optimal rules discussed above. The optimal responses to output growth and employment growth in the version with $W^{C} P^{C}$ are those that would make inflation invariant to demand and productivity shocks $(U$ and $X)$ in the version with $W^{F} P^{F}$. The solution for $\Pi^{*}$, inflation with $W^{F} P^{F}$, is given in equation (T1.1) in the Appendix, and $\Pi^{*}$ is invariant to $U$ and $X$ if the $\lambda_{i}$ are set at the values given in equation (91).

Suppose the policymaker can observe and respond only to output growth. With $W^{C} P^{F}$, when the policymaker observed a single variable, no matter which one, it could infer information about a combination of $U$ and $X$. For output growth with $W^{C} P^{C}$, the situation is different. The policymaker can infer the value of $U$ exactly but can learn nothing about $X$. The optimal response for the policymaker is to totally offset the effects of $U$ by strict targeting of $\Upsilon$ :

$$
\lambda_{\Upsilon} \rightarrow \infty, \quad \frac{\lambda_{\Pi}}{\lambda_{\Upsilon}}=\text { constant }, \quad \lambda_{\Lambda}=\lambda_{\Gamma}=\lambda_{U}=\lambda_{X}=\lambda_{Z}=0
$$

The resulting optimal log loss is

$$
\left.\ln \mathbb{L}\right|_{O} ^{\Upsilon}=\left(\frac{\tilde{\chi}}{\tilde{\alpha} J}\right)^{2} \sigma_{x}^{2}+\left(\frac{1}{J}\right)^{2} \sigma_{z}^{2}
$$

When all prices are set in contracts, nominal income growth targeting is clearly better than inflation targeting. Since inflation in predetermined, with inflation targeting the policymaker never responds to any shock. With predetermined inflation, nominal income growth targeting is the same as output growth targeting. Strict nominal income targeting is the best the policymaker can do with information about only inflation and output. All demand shocks are completely offset, but there is no response to productivity shocks because they do not lead to changes in output.

\footnotetext{
${ }^{39}$ In order to confirm the result stated in equation (91), divide the numerators and denominators of the coefficients of $\sigma_{u}^{2}$ and $\sigma_{x}^{2}$ by $\lambda_{\Lambda}$, let $\lambda_{\Lambda}$ and $\lambda_{\Upsilon}$ approach infinity holding the ratio $\frac{\lambda_{\Upsilon}}{\lambda_{\Lambda}}$ constant. Taking this limit drives the coefficient on $\sigma_{u}^{2}$ to zero. The coefficient on $\sigma_{x}^{2}$ can be set to zero by choosing the indicated value for $\frac{\lambda_{\Upsilon}}{\lambda_{\Lambda}}$.
} 
Now suppose that the policymaker can observe and respond only to employment growth. For this variable with $W^{C} P^{C}$ as for any single variable with $W^{C} P^{F}$, the policymaker can infer a combination of the $U$ and $X$ shocks. Strict employment growth targeting yields

$$
\left.\ln \mathbb{L}\right|_{S} ^{\Lambda}=\left(\frac{\tilde{\rho}}{J}\right)^{2} \sigma_{x}^{2}+\left(\frac{1}{J}\right)^{2} \sigma_{z}^{2}
$$

Strict employment growth targeting is better (worse) than strict output growth targeting when $\tilde{\rho}^{2} \lessgtr\left(\frac{\tilde{\chi}}{\tilde{\alpha}}\right)^{2}$. When $\tilde{\rho}^{2}$ is small, the full flexibility change in employment is small so that strict employment growth targeting is not very costly. However, if $\tilde{\rho}^{2}$ is large enough, strict output growth targeting is better.

Strict employment growth targeting is not the optimal response to information about employment growth. The optimal response to employment growth is

$$
\lambda_{\Lambda}=\psi_{L}+\frac{\rho \tilde{\chi}}{\tilde{\rho}}+\frac{J}{\rho \tilde{\rho}} \frac{\sigma_{u}^{2}}{\sigma_{x}^{2}}
$$

which implies

$$
\left.\ln \mathbb{L}\right|_{O} ^{\Lambda}=\left(\frac{\sigma_{u}^{2}}{\rho^{2} \sigma_{x}^{2}+\sigma_{u}^{2}}\right)\left(\frac{\tilde{\rho}}{J}\right)^{2} \sigma_{x}^{2}+\left(\frac{1}{J}\right)^{2} \sigma_{z}^{2}
$$

As in all earlier cases, the optimal response to employment growth is more aggressive the greater the relative importance of demand shocks, and the gap between the loss associated with strict targeting and the loss associated with the optimal response increases with the relative importance of the productivity shock.

It is interesting to compare optimal employment growth targeting and optimal nominal income growth targeting. When $(\tilde{\rho})^{2}<\left(\frac{\tilde{\chi}}{\tilde{\alpha}}\right)^{2}$, optimal employment targeting is clearly better. As shown above, in this case strict employment growth targeting is better than strict nominal income growth targeting. With $W^{C} P^{C}$, strict and optimal nominal income growth targeting are the same. By construction optimal employment targeting weakly dominates strict employment targeting. However, when $(\tilde{\rho})^{2}>\left(\frac{\tilde{\chi}}{\tilde{\alpha}}\right)^{2}$, the ranking depends on parameter values. For small $\sigma_{x}^{2}$, optimal nominal income growth targeting is better, since relatively more important demand shocks are completely offset under optimal nominal income growth targeting but not under optimal employment growth targeting.

\section{Conclusions}

In this paper we construct an optimizing-agent model with monopolistic competition in the product and labor markets and one-period nominal contracts. This model is simple enough that we can obtain exact solutions. We compare alternative simple monetary stabilization rules to optimal rules using as a criterion the expected utility of the representative agent. We focus on the two cases of (1) wage contracts and flexible prices and (2) wage and price contracts. 
Even in the presence of the distortions resulting from monopolistic competition, the best the monetary authority can do is to match the flexible price and wage equilibrium. In our model with one-period nominal contracts, the optimal rule under full information about the shocks can match the flexible price and wage equilibrium. We also calculate optimal rules under alternative assumptions about the partial information available to the policy maker. The optimal rules under partial information and simple rules can never achieve the full flexibility outcome because they imply no response to labor supply shocks. Of course, in general the optimal rule with full information dominates optimal rules with partial information which in turn dominate strict inflation targeting, strict nominal income growth targeting, and other simple rules. However, if there are no labor supply shocks, in a few special cases, optimal rules under partial information are as good as optimal rules under full information, and in even fewer special cases, some simple rules are as good as optimal rules under full information. Among suboptimal rules, nominal income growth targeting dominates inflation targeting for plausible parameter values.

A number of our conclusions regarding optimal rules under partial information and simple rules depend critically on the relative importance of productivity disturbances as measured by the ratio of the variance of the productivity disturbance to the variance of the demand disturbance. For example, the more important are productivity disturbances, (1) the lower the response coefficient in optimal rules based on observation of and response to a single variable, (2) the more suboptimal are strict inflation targeting, strict nominal income targeting, and other simple rules that involve total stabilization of a single variable rather than optimal response to that variable, and (3) the greater the advantage of nominal income growth targeting over inflation targeting. Another critical parameter is the elasticity of the disutility of labor (which, of course, is inversely related to the elasticity of labor supply). For example, if the elasticity of the disutility of labor is high, with wage contracts alone strict nominal income growth targeting performs very well, but with both wage and price contracts it performs very badly. 


\section{Appendix}

In this appendix, we summarize the properties of log normal distributions that are used in this paper and present tables containing the general solutions for the three versions of the model.

\section{Properties}

Suppose that the variable $Q$ has a log normal distribution; that is, suppose that $q=\ln Q \sim N\left(\mu_{q}, 2 \sigma_{q}^{2}\right)$. Now $\ln Q^{k}=k q$ so $Q^{k}=e^{k q}$. It follows that the $\mathcal{E}\left(Q^{k}\right)=$ $\mathcal{E}\left(e^{k q}\right)=M(q, k)$ where $M(q, k)$ is the moment generating function for $q$ and is given by

$$
M(q, k)=\int_{-\infty}^{\infty} e^{k q}\left[\frac{1}{2 \sqrt{\pi} \sigma_{q}} e^{-\frac{(q-\mu q)^{2}}{4 \sigma_{q}^{2}}}\right] d q=e^{k \mu_{q}+k^{2} \sigma_{q}^{2}}
$$

that is

$$
\mathcal{E}\left(Q^{k}\right)=e^{k \mu_{q}+k^{2} \sigma_{q}^{2}}
$$

Note that if $\mu_{q}=0$, then $\mathcal{E}(Q)=e^{\sigma_{q}^{2}} \neq 1$ and $\mathcal{E}\left(Q^{2}\right)=e^{4 \sigma_{q}^{2}}$. However, if $\mathcal{E}(Q)=1=$ $e^{\mu_{q}+\sigma_{q}^{2}}$, then $0=\mu_{q}+\sigma_{q}^{2}$ so $\mu_{q}=-\sigma_{q}^{2}$ and $\mathcal{E}\left(Q^{2}\right)=e^{2 \mu_{q}+4 \sigma_{q}^{2}}=e^{2 \sigma_{q}^{2}}$. We have assumed that $\mu_{q}=0$ in order to simplify our calculations. However, we can understand why others might prefer the alternative assumption.

Now suppose that the variables $U$ and $X$ are independently and log normally distributed; that is, suppose that $u=\ln U \sim N\left(\mu_{u}, 2 \sigma_{u}^{2}\right)$ and $x=\ln X \sim N\left(\mu_{x}, 2 \sigma_{x}^{2}\right)$. It follows that

$$
\mathcal{E}\left(U^{k_{U}} X^{k_{X}}\right)=e^{k_{U} \mu_{u}+k_{U}^{2} \sigma_{u}^{2}+k_{X} \mu_{x}+k_{X}^{2} \sigma_{x}^{2}} .
$$

\section{General Solutions}

The general solutions for the cases of full flexibility, wage contracts and flexible prices, and wage and price contracts are given in Tables 1, 2, and 3, respectively.

\begin{tabular}{|cc|}
\hline \multicolumn{3}{|c|}{ Table 1: Full Flexibility $\left(W^{F} P^{F}\right)$} & \\
$\Pi^{*}=\Phi Y_{-1}^{\phi_{Y}} L_{-1}^{\phi_{L}} U^{\phi_{U}} X^{\phi_{X}} Z^{\phi_{Z}}$, & $T 1.1$ \\
$\Omega^{*}=\Phi\left(\frac{s_{P}}{\theta_{P}}\right) Y_{-1}^{\phi_{Y}} L_{-1}^{\phi_{L}}\left(\frac{H}{D}\right)^{-\frac{\alpha}{J}} U^{\phi_{U}} X^{\phi_{X}+1+\frac{\alpha \tilde{\rho}}{J}} Z^{\phi_{Z}-\frac{\alpha}{J}}, \quad \phi_{Y}=\frac{\lambda_{\Upsilon}}{\lambda_{\Pi}}, \quad \phi_{L}=\frac{\lambda_{\Lambda}}{\lambda_{\Pi}}$, & $T 1.2$ \\
$\phi_{U}=\frac{1-\lambda_{U}}{\lambda_{\Pi}}, \quad \phi_{X}=-\frac{\lambda_{X} J+\tilde{\chi}\left(\lambda_{\Upsilon}-\phi_{Y}+\rho\right)-\tilde{\rho}\left(\lambda_{\Lambda}-\phi_{L}\right)}{\lambda_{\Pi} J}, \quad \phi_{Z}=-\frac{\lambda_{Z} J+\tilde{\alpha}\left(\lambda_{\Upsilon}-\phi_{Y}+\rho\right)+\left(\lambda_{\Lambda}-\phi_{L}\right)}{\lambda_{\Pi} J}$ & $T 1.3$ \\
$\ln \Phi=-\left(\frac{1}{\lambda_{\Pi}-1}\right) \ln \mathcal{E}\left(Q_{1}\right)-\left(\frac{\lambda_{\Upsilon}}{\lambda_{\Pi}}\right) \ln \left(\tilde{\alpha}^{-1}\left(\frac{H}{D}\right)^{\frac{\tilde{\alpha}}{J}}\right)-\left(\frac{\lambda_{\Lambda}}{\lambda_{\Pi} J}\right) \ln \left(\frac{H}{D}\right)$ & $T 1.4$ \\
$\ln \mathcal{E} Q_{1}=\left(1-\phi_{U}\right)^{2} \sigma_{u}^{2}+\left(\phi_{X}+\frac{\rho \tilde{\chi}}{J}\right)^{2} \sigma_{x}^{2}+\left(\phi_{Z}+\frac{\rho \tilde{\alpha}}{J}\right)^{2} \sigma_{z}^{2}$ & $T 1.5$ \\
\hline
\end{tabular}


Table 2: Wage Contracts and Flexible Prices $\left(W^{C} P^{F}\right)$

$$
\begin{aligned}
& L=\Xi U^{\xi_{U}} X^{\xi_{X}} Z^{\xi_{Z}} \\
& \xi_{U}=\frac{1-\lambda_{U}}{\Theta}, \xi_{X}=-\frac{\kappa}{\Theta}+\frac{\tilde{\chi} \lambda_{\Gamma}}{\Theta J}, \xi_{Z}=-\frac{\lambda_{Z}}{\Theta}+\frac{\tilde{\alpha} \lambda_{\Gamma}}{\Theta J}, \xi_{Y}=\frac{\lambda_{\Upsilon}}{\lambda_{\Pi}}, \xi_{L}=\frac{\lambda_{\Lambda}}{\lambda_{\Pi}} \\
& \kappa=\lambda_{X}+\rho+\lambda_{\Gamma}+\lambda_{\Upsilon}-\xi_{Y}-\lambda_{\Pi}, \Theta=\tilde{\alpha}\left(\rho+\lambda_{\Gamma}+\lambda_{\Upsilon}-\xi_{Y}\right)+\left(\lambda_{\Lambda}-\xi_{L}\right)+\alpha \lambda_{\Pi} \\
& \Xi=\left(\frac{H}{D} \frac{\mathcal{E}_{-1} Q_{2}}{\mathcal{E}_{-1} Q_{3}}\right)^{\frac{1}{J}}, \quad J=\tilde{\alpha} \tilde{\rho}+\tilde{\chi}, \quad A=\tilde{\alpha} \tilde{\rho}-\tilde{\chi} \\
& \ln \mathcal{E}_{-1} Q_{2}=\left(1-\xi_{U} \tilde{\alpha} \tilde{\rho}\right)^{2} \sigma_{u}^{2}+\left(\xi_{X} \tilde{\alpha}+1\right)^{2} \tilde{\rho}^{2} \sigma_{x}^{2}+\xi_{Z}^{2} \tilde{\alpha}^{2} \tilde{\rho}^{2} \sigma_{z}^{2} \\
& \ln \mathcal{E}_{-1} Q_{3}=\left(\xi_{U} \tilde{\chi}+1\right)^{2} \sigma_{u}^{2}+\xi_{X}^{2} \tilde{\chi}^{2} \sigma_{x}^{2}+\left(\xi_{Z} \tilde{\chi}-1\right)^{2} \sigma_{z}^{2} \\
& \ln \left(\frac{\mathcal{E}_{-1} Q_{2}}{\mathcal{E}_{-1} Q_{3}}\right)^{\frac{1}{J}}=\left(\xi_{U}^{2} A-2 \xi_{U}\right) \sigma_{u}^{2}+\left(\frac{\xi_{X}^{2} J A+\left(2 \xi_{X} \tilde{\alpha}+1\right) \tilde{\rho}^{2}}{J}\right) \sigma_{x}^{2}+\left(\frac{\xi_{Z}^{2} J A+2 \xi_{Z} \tilde{\chi}-1}{J}\right) \sigma_{z}^{2} \\
& \Omega=Y_{-1}^{\xi_{Y}} L_{-1}^{\xi_{L}} \bar{\Omega}, \quad \bar{\Omega}=\frac{s_{P}}{\theta_{P}}\left(\Xi^{\Theta-\rho \tilde{\alpha}-\alpha} \tilde{\alpha}^{-\left(\lambda_{\Upsilon}-\xi_{Y}\right)}\left(\frac{H}{D}\right)^{-\frac{\lambda_{\Gamma} \tilde{\alpha}}{J}} \mathcal{E} Q_{4}\right)^{-\frac{1}{\lambda_{\Pi}-1}} \\
& \ln \mathcal{E} Q_{4}=\left(\left(\frac{1}{\tilde{\alpha} \rho+\alpha}-\xi_{U}\right)^{2} \sigma_{u}^{2}+\left(\xi_{X}+\frac{\tilde{\rho}}{\tilde{\alpha} \rho+\alpha}\right)^{2} \sigma_{x}^{2}+\xi_{Z}^{2} \sigma_{z}^{2}\right)(\tilde{\alpha} \rho+\alpha)^{2}
\end{aligned}
$$


Table 3: Wage and Price Contracts $\left(W^{C} P^{C}\right)$

$$
\begin{aligned}
& L=\Psi U^{\psi_{U}} X^{\psi_{X}} Z^{\psi_{Z}} \\
& \psi_{U}=\frac{1-\lambda_{U}}{\digamma}, \psi_{X}=-\frac{\varepsilon}{\digamma}+\frac{\tilde{\chi} \lambda_{\Gamma}}{\digamma J}, \psi_{Z}=-\frac{\lambda_{Z}}{\digamma}+\frac{\tilde{\alpha} \lambda_{\Gamma}}{\digamma J}, \psi_{Y}=\frac{\lambda_{\Upsilon}}{\lambda_{\Pi}}, \psi_{L}=\frac{\lambda_{\Lambda}}{\lambda_{\Pi}} \\
& \varepsilon=\lambda_{X}+\rho+\lambda_{\Gamma}+\lambda_{\Upsilon}-\psi_{Y}, \quad \digamma=\tilde{\alpha}\left(\rho+\lambda_{\Gamma}+\lambda_{\Upsilon}-\psi_{Y}\right)+\left(\lambda_{\Lambda}-\psi_{L}\right) \\
& \Psi=\left(\frac{H}{D} \frac{\mathcal{E}_{-1} Q_{5}}{\mathcal{E}_{-1} Q_{6}}\right)^{\frac{1}{J}}, \quad J=\tilde{\alpha} \tilde{\rho}+\tilde{\chi}, \quad A=\tilde{\alpha} \tilde{\rho}-\tilde{\chi} \\
& \ln \mathcal{E}_{-1} Q_{5}=\left(\psi_{U} \tilde{\alpha} \tilde{\rho}-1\right)^{2} \sigma_{u}^{2}+\left(\psi_{X} \tilde{\alpha} \tilde{\rho}+\tilde{\rho}\right)^{2} \sigma_{x}^{2}+\psi_{Z}^{2} \tilde{\alpha}^{2} \tilde{\rho}^{2} \sigma_{z}^{2} \\
& \ln \mathcal{E}_{-1} Q_{6}=\left(\psi_{U} \tilde{\chi}+1\right)^{2} \sigma_{u}^{2}+\psi_{X}^{2} \tilde{\chi}^{2} \sigma_{x}^{2}+\left(\psi_{Z} \tilde{\chi}-1\right)^{2} \sigma_{z}^{2}, \\
& \ln \left(\frac{\mathcal{E}_{-1} Q_{5}}{\mathcal{E}_{-1} Q_{6}}\right)^{\frac{1}{J}}=\left(\psi_{U}^{2} A-2 \psi_{U}\right) \sigma_{u}^{2}+\left(\frac{\psi_{X}^{2} A+\left(2 \psi_{X} \tilde{\alpha}+1\right) \tilde{\rho}^{2}}{J}\right) \sigma_{x}^{2}+\left(\frac{\psi_{Z}^{2} A+2 \psi_{Z} \tilde{\chi}-1}{J}\right) \sigma_{z}^{2} \\
& \Pi=Y_{-1}^{\psi_{Y}} L_{-1}^{\psi_{L}} \bar{\Pi}, \quad \bar{\Pi}=\left(\Psi^{\tilde{\alpha}\left(\lambda_{\Gamma}+\lambda_{\Upsilon}-\psi_{Y}\right)+\left(\lambda_{\Lambda}-\psi_{L}\right)} \tilde{\alpha}^{-\left(\lambda_{\Upsilon}-\psi_{Y}\right)}\left(\frac{H}{D}\right)^{-\frac{\lambda_{\Gamma} \tilde{\alpha}}{J}} \mathcal{E} Q_{7}\right)^{-\frac{1}{\lambda_{\Pi}-1}} \\
& \ln \mathcal{E} Q_{7}=\left(1-\psi_{U} \tilde{\alpha} \rho\right)^{2} \sigma_{u}^{2}+\left(\psi_{X} \tilde{\alpha} \rho+\rho\right)^{2} \sigma_{x}^{2}+\psi_{Z}^{2} \tilde{\alpha}^{2} \rho^{2} \sigma_{z}^{2} \\
& \Omega=\Pi\left(\frac{s_{P}}{\theta_{P}}\right) \mathcal{E}_{-1}\left(\frac{U}{Y^{\tilde{\rho}}}\right)\left(\mathcal{E}_{-1}\left(\frac{L^{\alpha} U}{Y^{\tilde{\rho}} X}\right)\right)^{-1}=\Pi\left(\frac{s_{P}}{\theta_{P}}\right) \tilde{\alpha}^{\rho} \Psi^{-\tilde{\alpha} \tilde{\rho} \mathcal{E}_{-1} Q_{5}}
\end{aligned}
$$




\section{References}

Bean, C. (1983) "Targeting Nominal Income: An Appraisal", The Economic Journal, 93, 806-819.

Benassy, J.-P. (1995) "Money and Wage Contracts in an Optimizing Model of the Business Cycle", Journal of Monetary Economics, 35, 303-315.

Benhabib, J., S. Schmitt-Grohe, and M. Uribe (2001) "Monetary Policy and Multiple Equilibria", American Economic Review, 91, 167-185.

Canzoneri, M. B., R. E. Cumby, and B. T. Diba (2001) "Is the Price Level Determined by the Needs of Fiscal Solvency?", American Economic Review, 91, 1221-1238.

Corsetti, G., and P. Pesenti (2001) "Welfare and Macroeconomic Interdependence", Quarterly Journal of Economics, 116, 421-445.

Devereux, M. B., and C. Engel (1998) "Fixed vs. Floating Exchange Rates: How Price Setting Affects the Optimal Choice of Exchange-Rate Regime", NBER Working Paper 6867, National Bureau of Economic Research.

Engel, C. (1999) "On the Foreign Exchange Risk Premium in Sticky-Price General Equilibrium Models", International Tax and Public Finance, 6, 491-505.

Erceg, C. J., D. W. Henderson, and A. T. Levin (2000) "Optimal Monetary Policy with Staggered Wage and Price Contracts", Journal of Monetary Economics, 46, $281-313$.

Frisch, H., and S. Staudinger (2002) "Inflation Targeting versus Nominal Income Targeting", Journal of Economics, forthcoming.

Goodfriend, M., and R. King (1997) "The New Neoclassical Synthesis and the Role of Monetary Policy", in NBER Macroeconomics Annual 1997, MIT Press, Cambridge, 233-283.

Henderson, D., and W. McKibbin (1993) "A Comparison of Some Basic Monetary Policy Regimes for Open Economies: Implications of Different Degrees of Instrument Adjustment and Wage Persistence", in Carnegie-Rochester Series on Public Policy, Volume 39, 221-317.

Henderson, D. W., and J. Kim (1999) "Exact Utilities under Alternative Monetary Rules in a Simple Macro Model with Optimizing Agents", International Tax and Public Finance, 6, 507-535.

Henderson, D. W., and J. Kim (2001) "The Choice of a Monetary Policy Reaction Function in a Simple Optimizing Model", in A. Leijonhufvud (ed.) Monetary Theory and Policy Experience, Macmillan, London, 122-168.

Ireland, P. N. (1996) "The Role of Countercyclical Monetary Policy", Journal of Political Economy, 104, 704-723. 
Ireland, P. N. (1997) "A Small Structural, Quarterly Model for Monetary Policy Evaluation", in Carnegie-Rochester Series on Public Policy, Volume 47, 83-108.

Jensen, H. (1999) "Targeting Nominal Income Growth or Inflation?", CEPR Working Paper 2341, Centre for Economic Policy Research.

Kalchbrenner, J. H., P. A. Tinsley, J. Berry, and B. Garrett (1977) "On Filtering Auxiliary Information in Short-run Monetary Policy", in Carnegie-Rochester Series on Public Policy, Volume 7, 39-91.

Kim, J. (1997) "Three Sources of Increasing Returns to Scale", Finance and Economics Discussion Series 1997-18, Federal Reserve Board.

Kim, J. (2000) "Constructing and Estimating a Realistic Optimizing Model of Monetary Policy", Journal of Monetary Economics, 45, 329-359.

Kim, J. (2002) "Functional Equivalence between Intertemporal and Multisectoral Investment Adjustment Costs", Journal of Economic Dynamics and Control, forthcoming.

Kim, J., and S. H. Kim (2002) "Spurious Welfare Reversals in International Business Cycle Models", Journal of International Economics, forthcoming.

King, R. G., and A. L. Wolman (1999) "What Should the Monetary Authority Do When Prices Are Sticky?", in J. Taylor (ed.) Monetary Policy Rules, The University of Chicago Press, Chicago, 349-398.

Koenig, E. F. (1996) "Targeting Nominal Income: A Closer Look", Economics Letters, $51,89-93$.

LeRoy, S. F., and R. N. Waud (1977) "Applications of the Kalman Filter in Short-Run Monetary Control", International Economic Review, 18, 195-207.

McCallum, B. T., and E. Nelson (1999a) "Nominal Income Targeting in an Openeconomy Optimizing Model", NBER Working Paper 6675, National Bureau of Economic Research.

McCallum, B. T., and E. Nelson (1999b) "Nominal Income Targeting in an Openeconomy Optimizing Model", Journal of Monetary Economics, 43, 553-578.

Obstfeld, M., and K. Rogoff (1998) "Risk and Exchange Rates", NBER Working Paper 6694, National Bureau of Economic Research.

Obstfeld, M., and K. Rogoff (2000) "New Directions for Stochastic Open Economy Models", Journal of International Economics, 50, 117-154.

Ohanian, L. E., A. H. Stockman, and L. Killian (1995) "The Effects of Real and Monetary Policy Shocks in a Business Cycle Model with Some Sticky Prices", Journal of Money Credit, and Banking, 27-2, 1209-1234. 
Rotemberg, J. J., and M. Woodford (1997) "An Optimization-Based Econometric Framework for the Evaluation of Monetary Policy", in NBER Macroeconomics Annual 1997, MIT Press, Cambridge, 297-346.

Rotemberg, J. J., and M. Woodford (1998) "An Optimization-Based Econometric Framework for the Evaluation of Monetary Policy: Expanded Version", NBER Technical Working Paper T0233, National Bureau of Economic Research.

Rotemberg, J. J., and M. Woodford (1999) "Interest-Rate Rules in an Estimated Sticky Price Model", in J. Taylor (ed.) Monetary Policy Rules, The University of Chicago Press, Chicago, 57-119.

Svensson, L. E. O. (1999) "Price Level Targeting vs. Inflation Targeting: A Free Lunch?", Journal of Money, Credit, and Banking, 31, 277-295.

Woodford, M. (1999) "Inflation Stabilization and Welfare", processed, Princeton University. 


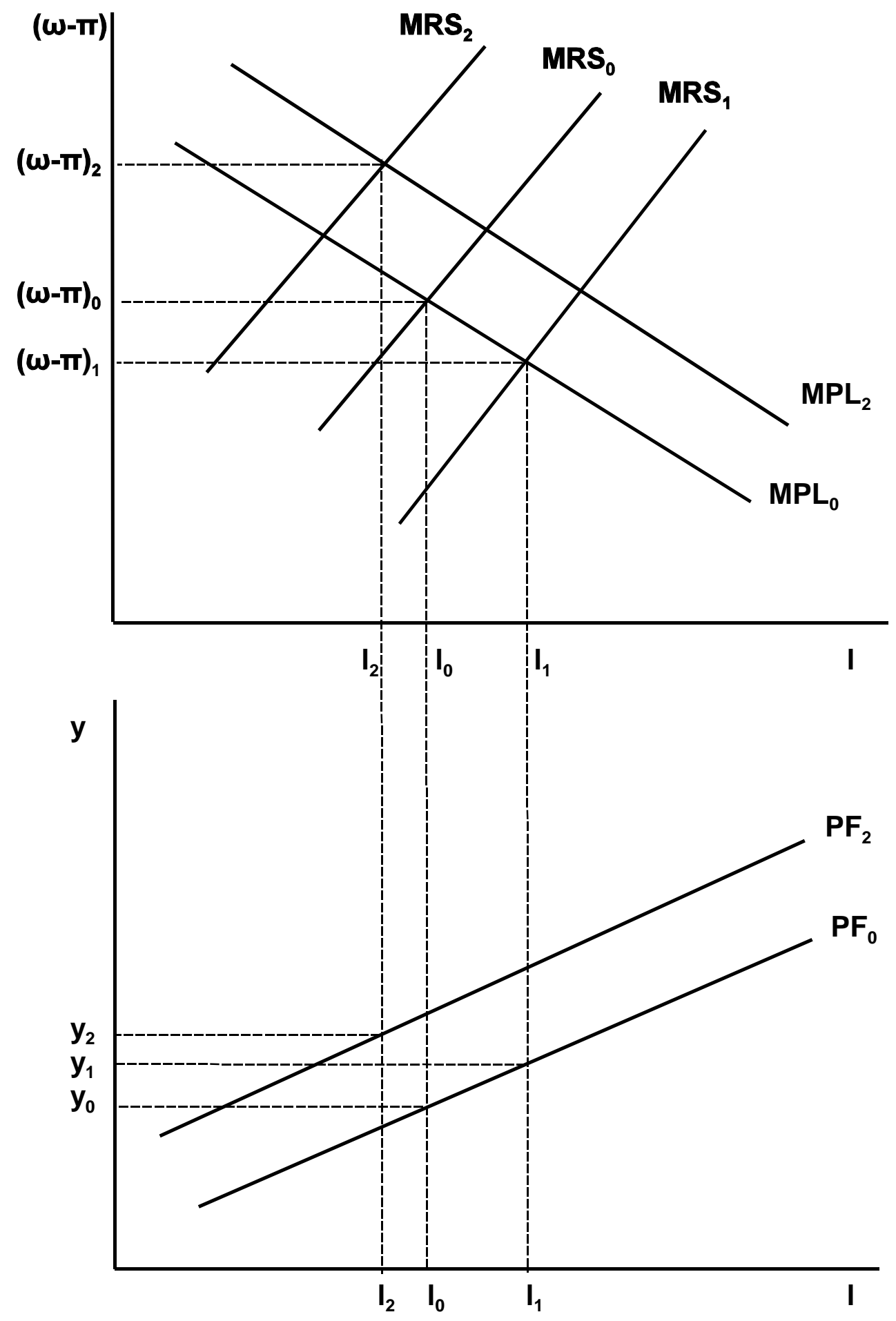

Figure 1. Flexible Wages and Prices 